\title{
Árboles útiles del bosque tropical caducifolio secundario en la Reserva Forestal Villa Allende, Chiapas, México
}

\section{Useful trees of the secondary deciduous tropical forest in Villa Allende Forest Reserve, Chiapas, Mexico}

\author{
Luis Alfredo Rodríguez Larramendi (D), María Silvia Sánchez Cortés² (D), Mercedes Concepción Gordillo Ruiz,4 (D)
}

1 Universidad de Ciencias y Artes de Chiapas, Coordinación de la Facultad de Ingeniería, Sede Villa Corzo, ejido Monterrey km 3, 30527 Villa Corzo, Chiapas, México.

2 Universidad de Ciencias y Artes de Chiapas, Instituto de Ciencias Biológicas, Libramiento Norte Poniente 1150, 29039 Tuxtla Gutiérrez, Chiapas, México

3 Universidad de Ciencias y Artes de Chiapas, Facultad de Ingeniería, Libramiento Norte Poniente 1150, 29039 Tuxtla Gutiérrez, Chiapas, México.

4 Autor para la correspondencia: marip_10@yahoo.mx

Recibido: 16 de marzo de 2018 Revisado: 11 de abril de 2018.

Aceptado: 7 de junio de 2018.

Primero en línea: 16 de agosto de 2018

Publicado: 3 de octubre de 2018.

Citar como:

Rodríguez Larramendi, L. A., M. S. Sánchez Cortés y M. C. Gordillo Ruiz. 2018. Árboles útiles del bosque tropical caducifolio secundario en la Reserva Forestal Villa Allende, Chiapas, México Acta Botanica Mexicana 125: 189-214. DOI: 10.21829/abml25.2018.1359

DOI:

10.21829/abml25.2018.1359

\section{Resumen:}

Antecedentes y Objetivos: El bosque tropical caducifolio (BTC) destaca por su valor utilitario, pero la información sobre el uso de las plantas por la población mestiza es limitada. Este estudio describe el valor de uso e importancia ecológica de las especies arbóreas nativas del BTC secundario cercano a comunidades campesinas en la Reserva Forestal Villa Allende, Chiapas, México.

Métodos: La información etnobotánica se obtuvo por la técnica de lista libre y se complementó con un inventario florístico a través del método de parcelas. Se calculó el índice de valor de uso como medida de la importancia cultural y se relacionó con la importancia ecológica de cada especie útil, usando valores relativos de frecuencia, densidad y dominancia.

Resultados clave: Se registraron 103 especies útiles de angiospermas, 77\% de ellas son de uso múltiple y corresponden a 10 categorías de uso, siendo los más destacados: combustible (73), postes (68) y construcción (63). Eysenhardtia adenostylis (1.36) y Acacia pennatula (1.29) fueron las especies con el valor de uso más alto, aunque la mayoría $(84 \%)$ registró bajos valores de uso $(\leq 0.3)$. Asimismo, las especies con mayor valor de uso poseen bajos valores de importancia ecológica (IVI $\leq 0.69$ ). El valor de uso por especie no se relacionó con el valor de importancia ecológica, excepto porque la dominancia del bosque de 19 años de abandono se correlacionó positivamente con el valor de uso $\left(\mathrm{r}_{\mathrm{s}}=0.30, \mathrm{P}=0.04\right)$ y la relación significativa entre la densidad con el valor de uso ornamental $\left(r_{s}=0.41, P=0.03\right)$. Con base en el valor de uso se recomendaron nueve especies potenciales para conservación y restauración del BTC en la zona estudiada.

Conclusiones: El BTC secundario en la Reserva Forestal Villa Allende, Chiapas, provee de especies arbóreas útiles para satisfacer las necesidades básicas de las comunidades, aunque la importancia cultural de éstas no depende de su importancia ecológica.

Palabras clave: área natural protegida, bosque secundario, comunidades mestizas, conocimiento tradicional, importancia ecológica, valor de uso.

\section{ABSTRACT:}

Background and Aims: The tropical deciduous forest (TDF) stands out for its utilitarian value, although the information related to the use of plants by mestizo communities is limited. This research describes the use value and the ecological importance of native TDF secondary tree species near peasant communities in Villa Allende Forest Reserve, Chiapas, Mexico.

Methods: The ethnobotanical information was obtained via the free list technique complemented with a floristic inventory through the method of plots. The use value index was calculated as a measure of cultural importance and it was related to the ecological importance value of each useful species, using relative values of frequency, density and dominance.

Key results: one hundred three useful angiosperm species were found, $77 \%$ of them are of multiple use and correspond to 10 categories in use, being the most remarkable: fuel (73), poles (68) and construction (63). Eysenhardtia adenostylis (1.36) and Acacia pennatula (1.29) were the species with the highest use value, although the majority (84\%) registered low use values $(\leq 0.3)$. Likewise, the species with the highest use value have low values of ecological importance (IVI $\leq 0.69)$. The use value per species was not related to the ecological importance value, except the dominance associated with the forest of 19 years of abandonment that correlated positively with the use value $\left(r_{s}=0.30, P=0.04\right)$ and the significant correlation between density and value of ornamental use $\left(\mathrm{r}_{\mathrm{s}}=0.41, \mathrm{P}=0.03\right)$. Based on use value, nine potential species were recommended for conservation and restoration of TDF in the study area.

Conclusions: The secondary TDF in Villa Allende Forest Reserve, Chiapas, provides useful tree species to satisfy the basic needs of the communities, although the cultural importance of these does not depend on its ecological importance.

Key words: ecological importance, mestizo communities, protected natural area, secondary forest, traditional knowledge, use value. 


\section{INTRODUCCIÓN}

El bosque tropical seco (BTS) destaca a nivel mundial por la riqueza biológica y el elevado número de endemismos que resguarda (Janzen, 1988). También se reconoce como un importante proveedor de recursos tangibles o materias primas (frutos, medicinas, leña, resinas, madera y combustibles) fundamentales en los medios de subsistencia de millones de personas alrededor del mundo (Salafsky y Wollenberg, 2000; Balvanera et al., 2011; Hickey et al., 2016). A pesar de su amplia distribución en América, África y Asia (aproximadamente 1,048,700 km²) (Miles et al., 2006), cerca de $97 \%$ de su superficie está en grave riesgo debido a diferentes actividades humanas (Murphy y Lugo, 1995; Miles et al., 2006; Holz y Placci, 2008), de forma que se le considera un ecosistema terrestre críticamente amenazado (Janzen, 1988; Vieira y Scariot, 2006; Quesada et al., 2009). La fragmentación, degradación y eliminación del bosque afecta las formas en que los seres humanos se interrelacionan con este ecosistema (Maffi, 2005; Díaz et al., 2006). Por lo tanto, la comprensión de estas relaciones puede contribuir en el diseño e implementación de estrategias de conservación de los bosques secos (Berkes et al., 2000).

La relación entre las personas y los bosques se ha analizado desde diferentes enfoques; no obstante, en las últimas dos décadas se ha incrementado el interés científico por estimar el significado o valor de uso de los bosques con el objetivo de identificar especies relevantes que muestren la interacción dinámica entre las personas y las plantas (Reyes-García et al., 2006). Entre los diversos trabajos se encuentran aquellos bajo un enfoque cultural, que consideran aspectos como los usos, propiedades utilitarias y preferencias de uso (Turner, 1988; Garibaldi y Turner, 2004) y otros con enfoque ecológico (Phillips y Gentry, 1993) que describen la importancia de las especies usadas a partir de la frecuencia de mención de las ecológicamente dominantes, en concordancia con la hipótesis de la apariencia ecológica (sensu Feeny, 1976). En este aspecto, las evidencias empíricas en el contexto del BTS, muestran que la relación entre el uso local de las especies y su importancia ecológica (representada por pa- rámetros estructurales) (Albuquerque y Lucena, 2005) es parcial y no siempre sigue un mismo patrón. Por ejemplo, Lucena et al. (2012) reportaron una correlación positiva entre la dominancia relativa y valor de uso de plantas leñosas ( $\geq 3 \mathrm{~cm}$ de DAP) utilizadas en la construcción rural en la comunidad de Cachoeira, y una correlación positiva entre el valor de uso general con el área basal y la dominancia relativa en la comunidad de Barrocas, ambas en la región de Catinga al noroeste de Brasil. Por su parte, Maldonado et al. (2013) encontraron una relación positiva entre el valor de uso de las especies leñosas $(>1 \mathrm{~cm}$ de DAP) y el valor de importancia ecológica (densidad y dominancia) de las plantas medicinales y alimenticias de las localidades indígenas y mestizas en la cuenca del Río Balsas, México. Por lo tanto, en términos de la conservación de la biodiversidad del BTS, es necesario identificar la posible forma en que la importancia ecológica de las especies del bosque se vincula con el uso local. Ambos aspectos pueden no solo ayudar a comprender los actuales patrones de uso, sino que también permitirían apoyar acciones de restauración y conservación.

Particularmente México posee una amplia variedad de bosques secos, propios de las regiones cálidas, agrupados bajo la denominación de bosque tropical caducifolio (BTC, sensu Rzedowski, 1978). La riqueza biológica que resguarda el BTC (Trejo y Dirzo, 2002) se suma a la riqueza cultural presentada en diversos estudios etnobotánicos que documentan la relación biocultural de cerca de la mitad de los grupos étnicos del país, quienes por largo tiempo han aprovechado ampliamente los productos del BTC, principalmente para la obtención de materiales de construcción, medicinas y combustibles (Bye, 1995; Zárate, 1999; Balvanera y Maass, 2010). Lamentablemente, en México la superficie del BTC se ha reducido dramáticamente, pasando de 35 millones de hectáreas de bosque en condición primaria en 2002 a 7.93 millones en 2008 (INEGI, 2003). Debido a esta situación, se trata del ecosistema con el mayor peligro de desaparecer a causa de la extensión de la frontera agropecuaria y la urbanización (Challenger y Soberón, 2008). En este mismo escenario de degradación se encuentra el BTC de la Reserva 
Forestal Villa Allende, localizado al centro de la región fisiográfica de la Depresión Central de Chiapas (Mulleried, 1951). La reserva fue decretada en 1939, para proteger la cobertura forestal del centro del estado de Chiapas (Vargas et al., 2000), pero actualmente está conformada por un mosaico de tierras agrícolas, centros de población, vegetación secundaria y remanentes de bosque primario en zonas de poca accesibilidad (Huerta García et al., 2016). Aunque en el área persiste menos de $2 \%$ del BTC en condición primaria (Ceballos et al., 2010; Vaca et al., 2012), en términos florísticos la región es valiosa debido a que registra una alta diversidad beta (Rocha-Loredo et al., 2010) y sirve de hábitat a diversas especies endémicas y protegidas por la legislación mexicana (Espinosa-Jiménez et al., 2014). Además, en el aspecto cultural, desde la época prehispánica, los Zoques han sido habitantes del BTC, aunque a partir de la colonia fueron reubicados en distintas ocasiones. En la actualidad se localizan en 12 municipios de Chiapas, y en particular en tres de ellos se registra su relación con el BTC. Este es el caso de algunas localidades de Tuxtla Gutiérrez, Ocozocoautla y Copainalá (Lisbona, 2006; Cruz-Burguete y Almazán-Esquivel, 2008), que, aunque son de origen Zoque, ya en ninguna se habla su lengua y domina la población mestiza. Existen estudios etnobotánicos cualitativos que describen el uso tradicional de más de 320 especies de plantas por parte de las comunidades Zoques (no hablantes) de Tuxtla Gutiérrez, Chiapas (Isidro, 1997; Isidro y Farrera, 1999). Para la Reserva Villa Allende la información sobre el uso de las plantas en las comunidades mestizas es limitada (Isidro et al., 2006; Sánchez, 2014; Pérez-Muñoz, 2017) y en ningún estudio se comparan los patrones de uso con datos ecológicos provenientes de inventarios de campo.

Por lo antes expuesto, la presente investigación tuvo como objetivo describir el valor de uso de las especies arbóreas nativas que crecen en el bosque secundario de la reserva, y conocer la importancia ecológica representada por parámetros estructurales en las formas de aprovechar las especies con base en el valor de uso, para de esta manera recomendar especies con potencial para la conservación y restauración del BTC en el área protegida de Villa Allende. La hipótesis planteada se refiere a corroborar que la importancia ecológica de las especies arbóreas se correlaciona positivamente con su valor de uso.

\section{Materiales y Métodos}

\section{Área de estudio}

El estudio se realizó en las comunidades de Berriozábal, Plan de Ayala y Viva Cárdenas, las cuales se localizan en la porción suroeste de la Reserva Forestal Villa Allende entre los paralelos $16^{\circ} 47^{\prime} \mathrm{N}, 93^{\circ} 12^{\prime} \mathrm{O}, 16^{\circ} 48^{\prime} \mathrm{S}, 91^{\circ} 11^{\prime} \mathrm{E}$ (Fig. 1). El clima predominante es cálido con temperatura media cercana a $26{ }^{\circ} \mathrm{C}$ y precipitación promedio anual de $955.8 \mathrm{~mm}$ (López, 2006). El bosque tropical caducifolio secundario es la vegetación dominante en la zona, pero suele formar transiciones con bosque de encino y bosque tropical subcaducifolio (Espinosa-Jiménez et al., 2014). El bosque secundario generalmente se desarrolla entre los 500 y 1000 metros de altitud (INEGI, 1999), en suelos de tipo litosol (INEGI, 2000) y presenta elementos característicos del BTC, como Bursera Jacq. ex L. y Ficus L. (Rzedowski, 2006). No obstante, la composición y la estructura varían ampliamente dependiendo de la edad sucesional y del tipo de perturbación a la cual se encuentra sometida (Gordillo-Ruiz et al., datos no publicados).

\section{Descripción de las comunidades de estudio}

Las comunidades seleccionadas (Berriozábal, Plan de Ayala y Viva Cárdenas) poseen una tenencia de la tierra de régimen ejidal y en conjunto mantienen la zona boscosa más grande del BTC secundario de la Reserva Villa Allende, por lo que tienen un papel relevante para la conservación del bosque.

Berriozábal tiene una extensión de 4916.79 ha y se localiza entre $16^{\circ} 48^{\prime} \mathrm{N}, 93^{\circ} 16^{\prime} \mathrm{O}$, a una altitud de $900 \mathrm{~m}$. La mayor parte de la superficie ejidal (1925.29 ha) está destinada al uso agropecuario, 1338.03 ha corresponden a bosque secundario, 423.97 ha a bosque ripario y 1229.5 ha son parte de la zona urbana de la cabecera municipal Berriozábal. 


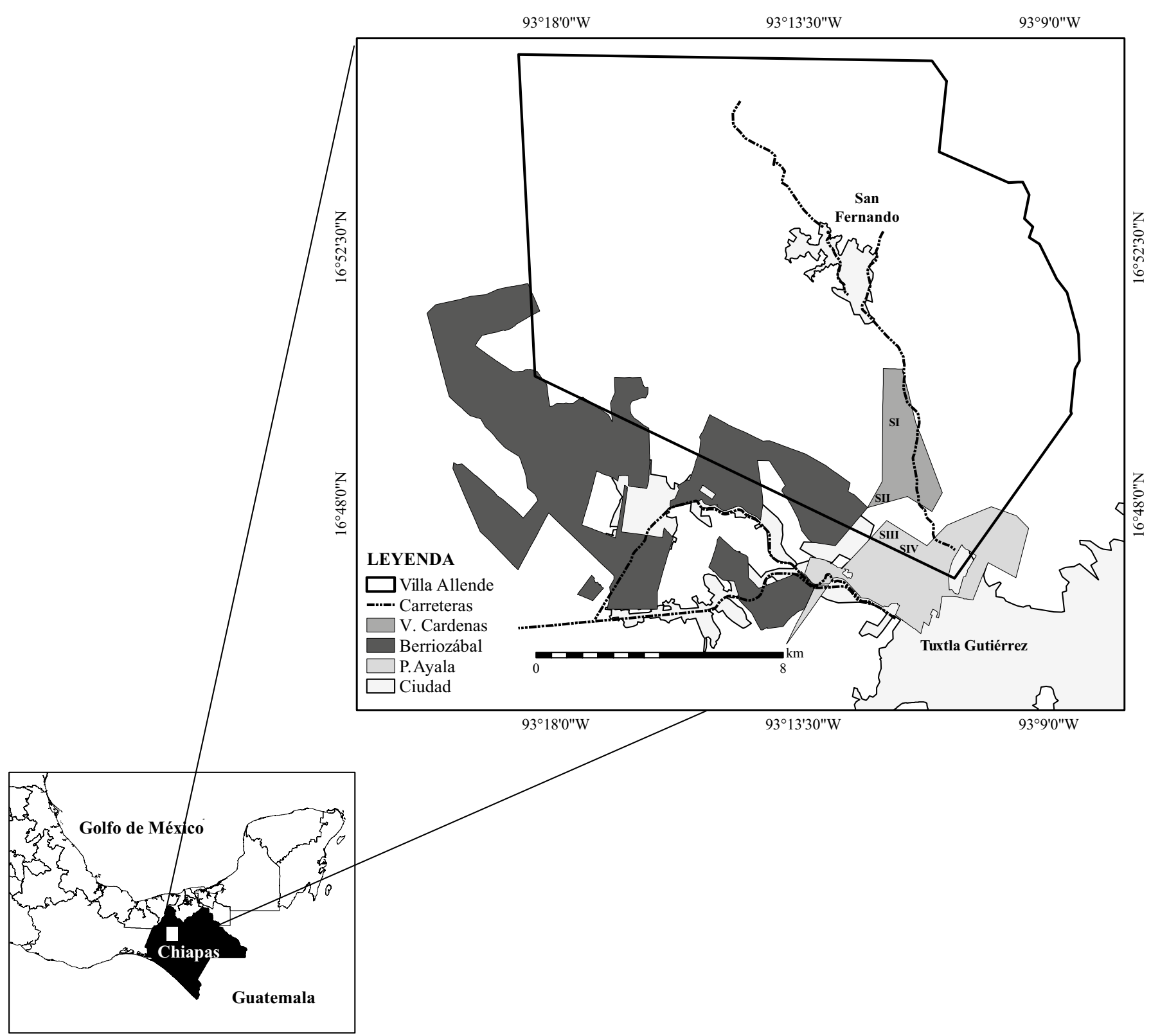

Figura 1: Localización de las comunidades de estudio en la Reserva Forestal Villa Allende, Chiapas, México, y los sitios de muestreo en campo (S).

Plan de Ayala está dentro del área urbana de la ciudad de Tuxtla Gutiérrez (capital del estado de Chiapas), entre $16^{\circ} 45^{\prime} \mathrm{N}, 93^{\circ} 10^{\prime} \mathrm{O}$, a una altitud de $594 \mathrm{~m}$. Posee una superficie ejidal de 1034.03 ha, distribuidas en 199.18 ha de uso agrícola, 648.8 ha de bosque secundario y 236.05 ha de zona urbana.

A diez kilómetros de Plan de Ayala se localiza Viva Cárdenas, del municipio San Fernando, entre $16^{\circ} 50^{\prime} \mathrm{N}$, $93^{\circ} 11^{\prime} \mathrm{O}$, a una altitud de $837 \mathrm{~m}$. Tiene una superficie de
565.6 ha, 261.54 ha destinadas a cultivos, 216.42 ha a bosque secundario y 87.54 ha a la zona urbana. Básicamente, en todos los ejidos, el bosque secundario se localiza dentro del área de uso común.

De acuerdo con INEGI (2010), Berriozábal posee una población de 28,128 habitantes (14,027 hombres y 14,101 mujeres), Plan de Ayala de 9045 (4567 hombres y 4478 mujeres) y Viva Cárdenas de 1431 (712 hombres y 719 mujeres). En términos agrarios, Berriozábal está inte- 
grado por 600 ejidatarios, pero solo $20 \%$ de los que se dedican a las actividades de campo poseen terrenos agrícolas. Plan de Ayala posee una agrupación de 138 ejidatarios, de los cuales solo $30 \%$ tienen parcelas agrícolas en el distrito de riego denominado San Agustín. En Viva Cárdenas existen 45 ejidatarios, pero solo 23 conservan terrenos agrícolas. En todas las localidades, los cultivos están contiguos a la zona de distribución del BTC secundario. El resto de la población ejidal mantiene sus derechos debido a que aún conservan zonas de uso común, que a excepción de Viva Cárdenas, que posee un banco de arena, se trata de terrenos forestales. El 90\% de la población ejidal es nativa. A excepción de Berriozábal, cuya población campesina combina actividades agrícolas y ganaderas, las otras dos comunidades se dedican al cultivo de maíz, frijol, café y frutales. Sin embargo, la venta de los productos agrícolas junto con actividades de servicios (p. ej. jornaleros, comerciantes o albañiles) constituyen sus principales ingresos.

\section{Inventario de las especies arbóreas}

Se seleccionaron cuatro sitios de bosque secundario, con características homogéneas de suelo, derivado del abandono de potreros de las distintas edades indicadas por los campesinos a través de entrevistas, y corroboradas con recorridos de campo, y con el apoyo de fotografías aéreas e imágenes satelitales en Google Earth.

El sitio I es un bosque con un tiempo de abandono de 10 años mezclado con elementos de la vegetación original característica del bosque tropical caducifolio, situado en el límite del área urbana de Viva Cárdenas. Los sitios II (19 años de abandono), III (35 años) y IV ( $>40$ años) se ubican en el remanente de bosque secundario más grande (2105.9 ha) dentro de la Reserva Villa Allende, en una zona de lomeríos que abarca los límites de los tres ejidos (Fig. 1). De 2014 a 2016, en cada sitio se establecieron parcelas de muestreo de la vegetación $(20 \times 50 \mathrm{~cm}, 0.1 \mathrm{ha})$, cinco en $\mathrm{I}$, siete en II y III, y tres en IV. La diferencia en el número de parcelas obedeció a que sitios con 10 y $>40$ años de abandono son una condición reducida en el área de estudio. Las parcelas se distribuyeron en zonas entre 800 a $1000 \mathrm{~m}$ de altitud, con suelos tipo litosol y pendientes ligeras.
De acuerdo con la propuesta de Kalacska et al. (2004), en cada parcela se identificó y midió el diámetro a la altura del pecho (DAP) de todos los individuos arbóreos de $\leq 1.30 \mathrm{~m}$ de altura y con $\mathrm{DAP} \geq 5 \mathrm{~cm}$. Las especies que no fueron identificadas en campo fueron colectadas y determinadas mediante el uso de claves especializadas y por comparación con ejemplares de las colecciones de los herbarios HEM (Eizi Matuda del Instituto de Ciencias Biológicas de la Universidad de Ciencias y Artes de Chiapas) y CHIP (Herbario de la Secretaria de Medio Ambiente e Historia Natural); una serie de ejemplares recolectados se depositó en estos herbarios. Las familias se ordenaron según la clasificación de Angiosperm Phylogeny Group (APG IV, 2016). Los nombres y las abreviaciones de las autoridades de los taxones se citaron siguiendo The International Plant Names Index (IPNI, 2012).

A partir del inventario de árboles se estimó la densidad, el área basal (dominancia) y la frecuencia de cada especie. Con estos valores se calculó el valor de importancia ecológica por especie, empleando la fórmula propuesta por Mueller-Dombois y Ellenberg (2002):

$\mathrm{IVI}=$ dominancia relativa + densidad relativa + frecuencia relativa/3.

\section{Valor de uso}

Los ejidatarios son los habitantes que poseen los derechos legales de los terrenos (agrícolas y forestales), y se consideran actores fundamentales de la historia, conocimiento, uso y destino de los recursos naturales en el núcleo ejidal (Salazar, 2000). Por ello, de octubre de 2014 a septiembre de 2017 se entrevistaron 111 personas con derechos ejidales señalados por los mismos campesinos como conocedores y usuarios de los árboles del ecosistema de estudio (Goodman, 1961; Moreno-Casasola y Paradowska, 2009). En el cuadro 1 se describe el perfil socioeconómico de los entrevistados en las comunidades estudiadas. La eficiencia en la colecta de los datos etnobotánicos se estimó a partir de una curva de acumulación de especies usando los estimadores no paramétricos de Chao 2, ICE, Jackknife 1, Jackknife 2 y Bootstrap (Williams et al., 2007). Para reco- 
Cuadro 1: Número, sexo, edad promedio (entre paréntesis la desviación estándar) y ocupación (agricultura (A), ganadería (G), servicios (SR) y agricultura o ganadería $(\mathrm{A} / \mathrm{G})$ ) de los participantes en las entrevistas etnobotánicas en las comunidades Berriozábal, Plan de Ayala y Viva Cárdenas. *Valores absolutos.

\begin{tabular}{lccccccccc}
\hline Localidad & \multicolumn{2}{c}{ Número de entrevistados } & \multicolumn{2}{c}{ Edad promedio } & \multicolumn{3}{c}{ Ocupaciówn } \\
\cline { 2 - 9 } & Hombres & Mujeres & Hombres & Mujeres & A & A+G & A/G+SR & G & SR \\
\hline Berriozábal & 50 & 1 & $62.2(10.8)$ & $60.0(0.0)^{*}$ & 25 & 7 & 15 & 3 & 1 \\
Plan de Ayala & 29 & 4 & $69.0(11.3)$ & $52.75(14.9)$ & 18 & 1 & 12 & 0 & 2 \\
Viva Cárdenas & 26 & 1 & $63.3(12.8)$ & $58.0(0.0)^{*}$ & 21 & 0 & 4 & 0 & 2 \\
\hline
\end{tabular}

pilar la información etnobotánica durante la entrevista se aplicó la técnica de lista libre (Reyes-García et al., 2006). En la primera parte de la entrevista se recabaron los datos personales de los entrevistados (nombre, edad, origen migratorio y actividad económica) y en la segunda parte se registró la lista de los árboles presentes en la comunidad y los usos atribuidos a cada uno.

La información de los usos locales de las especies se organizó en las siguientes categorías de uso: a) combustible (leña con fines domésticos e industriales), b) alimenticias (comestibles frecuentemente de productos tradicionales), c) medicinales (tratamiento de afectaciones a la salud con base en una modificación hecha a la Clasificación Internacional de las Enfermedades del Manual de la Clasificación Estadística Internacional de Enfermedades y Causas de Defunción (OPS, 1995) y la medicina tradicional (INI, 1994), d) maderable (elaboración de muebles, carretas, herramientas de trabajo, utensilios domésticos, entre otros), e) construcción (elaboración de partes de la vivienda rural), f) cerco vivo (aprovechadas con la finalidad de cercar sus huertos familiares y terrenos de cultivo), g) poste (delimitación de terrenos de uso común del ejido, parcelas agrícolas y potreros), h) forrajeras (alternativa para alimentar ganado en el periodo de estiaje), i) ornamentales (embellecimiento de parques locales y patios de las casas) y j) religioso (festividades religiosas).

A los entrevistados se les mostraron ejemplares botánicos del inventario de campo para confirmar las especies de los registros citados por ellos. No obstante, cuando se presentó discrepancia entre una u otra persona en cuan- to al nombre común, o se identificó un nuevo registro citado por los entrevistados pero no colectado en inventario, se realizó la colecta del ejemplar a través de caminatas en compañía del informante que mencionó el nombre local o de personas que demostraron tener mayor conocimiento durante la entrevista.

\section{Análisis de datos}

El valor de uso de cada especie a nivel general y de las categorías de uso se estimó a partir de la frecuencia de mención indicada por los entrevistados. Para ello se empleó el índice de valor de uso propuesto por Phillips y Gentry (1993), y modificado por Rossato et al. (1999), usando las siguientes fórmulas:

$$
\mathrm{VU}=\Sigma U s i n^{-1}
$$

VU es el valor de uso global para cada especie, donde $\mathrm{VU}$ es igual al número de usos mencionados en cada entrevista para la especie $U s$ por informante $i$, y $n$ el número total de informantes entrevistados en el muestreo.

\section{$\mathrm{VUc}=\Sigma U c i . n^{-1}$}

El VUc es el valor de uso a nivel de categoría, donde $U c$ representa el número de usos atribuidos a una especie listada dentro de cada categoría de uso por informante $i$ y $n$ el número total de informantes.

Por otra parte, para establecer la posible relación entre la importancia ecológica de las especies arbóreas y el valor 
de uso, se emplearon los valores relativos de abundancia, frecuencia, dominancia y el índice valor de importancia. Debido a la falta de normalidad de los datos se optó por realizar un análisis de correlación de Spearman. Las especies registradas en el inventario de campo, pero no usadas por las personas, así como las nombradas pero ausentes en el inventario, no fueron consideradas en el análisis. Las correlaciones se efectuaron considerando los valores de la importancia ecológica general (IVIg) y por condición del bosque (representada por la edad de abandono) comparándose con el valor de uso global a nivel de especie y entre el IVIg con el valor de uso en cada categoría de uso. Los análisis estadísticos se realizaron en el programa $\mathrm{R}$ versión 3.1.2. (R Core Team, 2013).

\section{Resultados}

\section{Usos de las especies arbóreas}

Los entrevistados mencionaron 102 especies útiles de angiospermas, distribuidas en 77 géneros y 37 familias
(Apéndice). La familia Fabaceae reportó el mayor número de especies (29), seguida por Malvaceae y Anacardiaceae con cinco especies cada una. También se nombraron 17 especies de origen exótico, pero éstas no se incluyeron en el análisis debido a que el interés del estudio se centró en las especies nativas. De acuerdo con el análisis de acumulación de especies, el esfuerzo en su registro representó entre 69\% (Jackknife 2) y 90\% (Bootstrap) de las 132 que se esperaría encontrar (Fig. 2). De forma complementaria, la curva de especies mencionadas una sola vez (singletons) no declinó sobre la curva de especies con dos menciones (doubletons), sugiriendo que aún es posible registrar más especies.

Los taxones registrados se clasificaron en 10 categorías de uso (Cuadro 2). De éstos, 21 especies registraron solo un uso y el resto usos múltiples (Apéndice). Las categorías más importantes en función del número de especies mencionadas fueron: combustible $(18.6 \%$ de todas las especies útiles), poste $(66,17.0 \%)$ y construc-

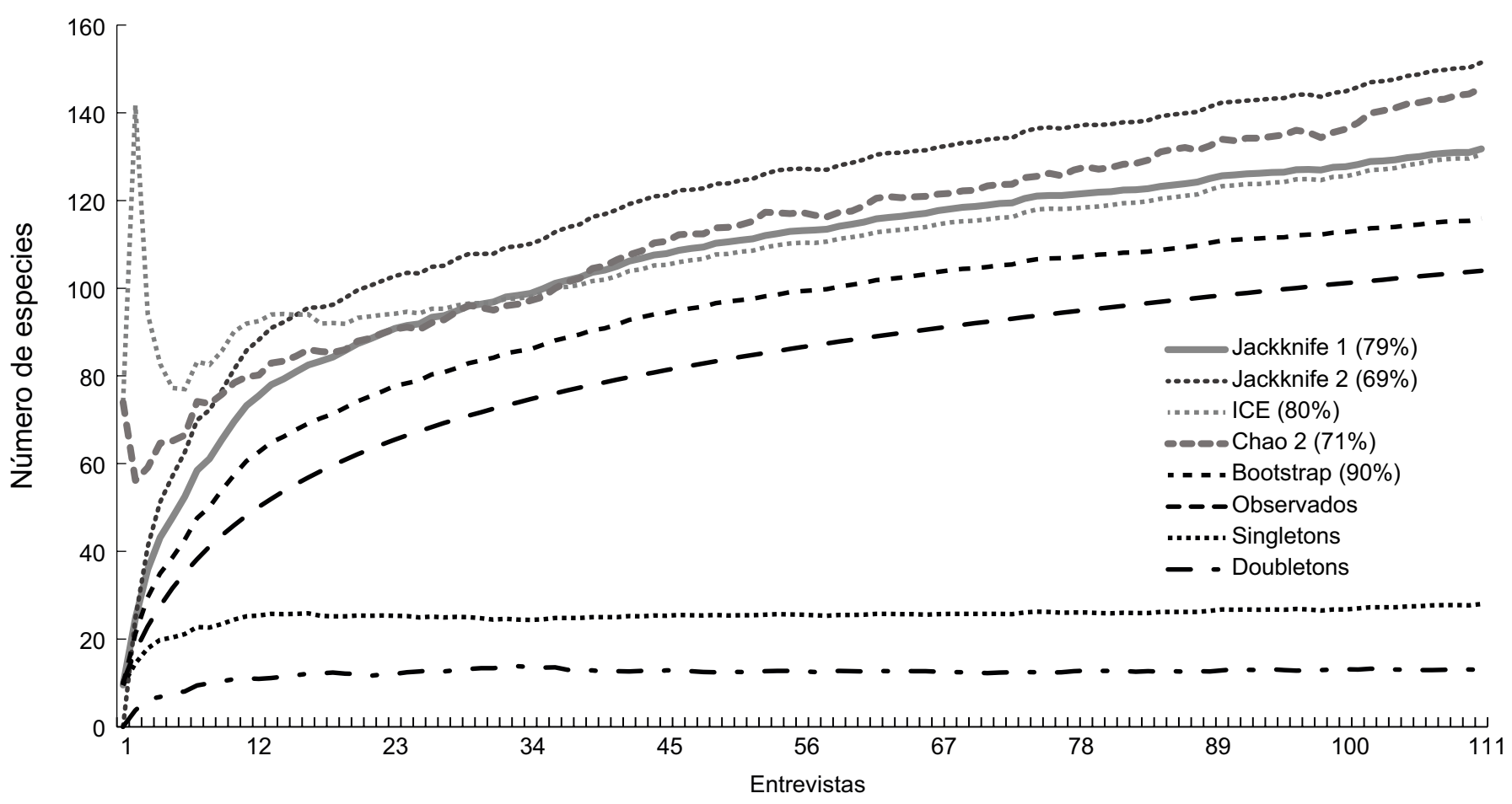

Figura 2: Curva de acumulación de especies mencionadas por los entrevistados en tres comunidades al sur de la Reserva Forestal Villa Allende, Chiapas. Observados: especies mencionadas por los entrevistados; Jackknife 1, Jackknife 2, ICE, Chao 2 y Bootstrap: estimadores no-paramétricos de la riqueza de especies. Singletons: número de especies mencionadas una vez; Doubletons: número de especies mencionadas dos veces. Entre paréntesis se presenta el porcentaje de especies registrado en las 111 entrevistas, respecto de aquel esperado por los estimadores no-paramétricos. 
ción rural $(63,16.2 \%)$, seguidas por el uso medicinal (43, $11.1 \%)$ y maderable $(39,10.1 \%)$. Dentro de las categorías de uso evaluadas, algunas (7) registraron subcategorías de uso (22) y usos específicos (78) (Cuadro 3). De las especies arbóreas medicinales $43.4 \%$ se emplearon en el tratamiento de problemas gastrointestinales, seguidas de las enfermedades propias del sistema músculo-esquelético (13.2\%) y del sistema urinario (10.5\%), y en menor proporción aquellas que contribuyen a atenuar problemas relacionados con el sistema circulatorio y respiratorio ( $7.9 \%$ y $6.6 \%$ respectivamente). Del total de especies usadas para la construcción rural, la mayor parte de las especies se aprovecharon para obtener horcones (25\%), seguidas del uso de morillo (21.7\%), solera (16.3\%) y tijera (12\%); los usos restantes registraron valores menores a $8 \%$. Asimismo, en la categoría de maderables $34.9 \%$ de las especies se usaron en la fabricación de muebles del hogar, 33.7\% en la elaboración de herramientas de trabajo y $26.7 \%$ se aprovecharon en las diferentes partes de la estructura de la carreta y en la fabricación de muebles. De las especies usadas como alimento, $65 \%$ fueron apreciadas por sus frutos y en menor medida en la preparación de bebidas y como condimento de platillos típicos $(10 \%$ en cada caso). El resto de las subcategorías no presentaron amplias diferencias en cuanto al número de especies empleadas en cada uso específico.

\section{Valor de uso y Valor de importancia ecológica}

De las especies mencionadas por los entrevistados, $65 \%$ fueron registradas en el inventario de campo (Apéndice). De éstas, los valores de uso (VU) más altos los registraron Eysenhardtia adenostylis Baill. (1.36) y Acacia pennatula (Schltdl. \& Cham.) Benth. (1.29). Eysenhardtia adenostylis es una especie reportada en todas las categorías de uso, mientras $A$. pennatula se utilizó en las categorías de construcción rural, combustible, forraje, poste y cerco vivo (Apéndice). Dieciséis especies multipropósito mostraron un valor de uso intermedio (0.34-0.97) y el resto (84.6\%) reportó valores menores a 0.3 . Las especies con el mayor VU registraron bajos valores de importancia ecológica (IVI=0.27-0.69, Apéndice). Del total de es-
Cuadro 2: Número de especies y proporciones (incluye especies con más de un uso $n=388 \mathrm{spp}$.) por categoría de uso en las comunidades de estudio de la Reserva Forestal Villa Allende, Chiapas, México.

\begin{tabular}{lcc}
\hline Categoría de uso & Número de especies & Proporción \\
\hline Combustible & 72 & 18.6 \\
Poste & 66 & 17.0 \\
Construcción rural & 63 & 16.2 \\
Medicinal & 43 & 11.1 \\
Maderable & 39 & 10.1 \\
Ornamental & 30 & 7.7 \\
Alimento & 24 & 6.2 \\
Cerco vivo & 22 & 5.7 \\
Forrajera & 18 & 4.6 \\
Religioso & 11 & 2.8 \\
\hline
\end{tabular}

pecies con VU intermedio, solo Bursera simaruba (L.) Sarg., Lysiloma acapulcensis (Kunth) Benth. y Alvaradoa amorphoides Liebm. reportaron un IVI alto $(\geq 1)$. De las especies con $\mathrm{VU}<0.3$, Cochlospermum vitifolium (Willd.) Spreng., Heliocarpus terebinthinaceus (DC.) Hochr., Luehea candida (Moc. \& Sessé ex DC.) Mart., Machaerium arboreum (Jacq.) Vogel, Montanoa tomentosa Cerv., Perymenium grande Hemsl. y Tecoma stans (L.) Juss. ex Kunth mostraron altos valores de IVI (1.9-3.4).

\section{Valor de uso por categoría}

Veinticuatro especies registraron los valores de uso más altos en las 10 categorías de uso, las cuales fueron empleadas para satisfacer 72 de los 78 usos específicos. En la categoría de combustible destacan las especies Acacia pennatula $(\mathrm{VUc}=0.82)$, Leucaena collinsii Britton \& Rose $(\mathrm{VUc}=0.39)$ y Eysenhardtia adenostylis ( $\mathrm{VUc}=0.30)$, utilizadas específicamente como leña para uso doméstico. Estas mismas especies, junto con Colubrina arborescens Sarg., fueron las más valoradas en la categoría de poste (VUp $=0.47,0.52,0.64$ y 0.38 respectivamente). De igual manera, en la categoría de construcción rural Eysenhardtia adenostylis (VUct=0.71), generalmente usada para horcón y solera, seguida de Colubrina arborescens (VUct=0.65), 
Cuadro 3: Subcategorías y usos específicos dentro de las categorías de uso de las especies arbóreas de tres comunidades de la Reserva Forestal Villa Allende, Chiapas, México. La proporción incluye especies con más de un uso (medicinal=76, alimento $=20$, maderable $=86$, combustible $=81$, construcción $=92$, religioso $=10$ y forrajera $=25)$.

\begin{tabular}{|c|c|c|c|c|}
\hline Categoría & Sub-categoría & Uso específico & No. Especies & Proporción \\
\hline \multirow[t]{30}{*}{ Medicinal } & \multirow[t]{3}{*}{ Sistema circulatorio } & heridas & 3 & 3.9 \\
\hline & & anemia & 2 & 2.6 \\
\hline & & hemorragia & 1 & 1.3 \\
\hline & Sistema nervioso & dolor de cabeza & 2 & 2.6 \\
\hline & \multirow[t]{11}{*}{ Sistema gastroinstestinal } & diarrea & 7 & 9.2 \\
\hline & & diabetes & 6 & 7.9 \\
\hline & & dolor de estómago & 5 & 6.6 \\
\hline & & gastritis & 5 & 6.6 \\
\hline & & dolor de muela & 2 & 2.6 \\
\hline & & desinflamar vesícula & 2 & 2.6 \\
\hline & & estreñimiento & 2 & 2.6 \\
\hline & & úlcera & 1 & 1.3 \\
\hline & & colitis & 1 & 1.3 \\
\hline & & hemorroides & 1 & 1.3 \\
\hline & & depurativa & 1 & 1.3 \\
\hline & \multirow[t]{4}{*}{ Sistema músculo-esquelético } & inflamación de los órganos & 5 & 6.6 \\
\hline & & golpes & 3 & 3.9 \\
\hline & & dolor muscular & 1 & 1.3 \\
\hline & & dolor de huesos & 1 & 1.3 \\
\hline & \multirow[t]{2}{*}{ Sistema respiratorio } & gripa & 4 & 5.3 \\
\hline & & tos & 1 & 1.3 \\
\hline & \multirow[t]{2}{*}{ Sistema urinario } & cálculos renales & 4 & 5.3 \\
\hline & & inflamación de la próstata & 4 & 5.3 \\
\hline & Sistema reproductivo & regular la menstruación & 1 & 1.3 \\
\hline & Sistema linfático & depurativa & 2 & 2.6 \\
\hline & Sistema tegumentario & ampollas & 1 & 1.3 \\
\hline & \multirow[t]{2}{*}{ Infecciones } & parásitos & 3 & 3.9 \\
\hline & & sarampión & 1 & 1.3 \\
\hline & \multirow[t]{2}{*}{ Enfermedades místicas } & mal de aire & 3 & 3.9 \\
\hline & & mal de espanto & 1 & 1.3 \\
\hline \multirow[t]{4}{*}{ Alimento } & \multirow[t]{4}{*}{ Comestible } & frutas & 13 & 65.0 \\
\hline & & bebida & 2 & 10.0 \\
\hline & & condimento & 2 & 10.0 \\
\hline & & dulces & 1 & 5.0 \\
\hline
\end{tabular}


Cuadro 3: Continuación.

\begin{tabular}{|c|c|c|c|c|}
\hline Categoría & Sub-categoría & Uso específico & No. Especies & Proporción \\
\hline & & tamales & 1 & 5.0 \\
\hline & & guisado & 1 & 5.0 \\
\hline \multirow[t]{22}{*}{ Maderable } & Estructura de carreta & eje & 10 & 11.6 \\
\hline & & masa & 4 & 4.7 \\
\hline & & larguero & 3 & 3.5 \\
\hline & & timón & 3 & 3.5 \\
\hline & & rayo & 2 & 2.3 \\
\hline & & cercha & 1 & 1.2 \\
\hline & Herramientas domésticas & escoba & 1 & 1.2 \\
\hline & Herramientas de trabajo & yugo & 14 & 16.3 \\
\hline & & montura & 4 & 4.7 \\
\hline & & cabo de coa & 4 & 4.7 \\
\hline & & timón arado & 4 & 4.7 \\
\hline & & cabo de hacha & 2 & 2.3 \\
\hline & & escalera & 1 & 1.2 \\
\hline & Muebles & mesa & 10 & 11.6 \\
\hline & & silla & 10 & 11.6 \\
\hline & & cama & 6 & 7.0 \\
\hline & & puerta & 2 & 2.3 \\
\hline & & esquinero & 1 & 1.2 \\
\hline & & colgador & 1 & 1.2 \\
\hline & Otros & canoa & 1 & 1.2 \\
\hline & & juguete & 1 & 1.2 \\
\hline & & reja & 1 & 1.2 \\
\hline \multirow[t]{3}{*}{ Combustible } & Uso doméstico & leña & 73 & 90.1 \\
\hline & Uso industrial & cocimiento de panela & 7 & 8.6 \\
\hline & & cocimiento de ladrillo & 1 & 1.2 \\
\hline \multirow[t]{8}{*}{ Construcción rural } & Partes de la vivienda & horcón & 23 & 25.0 \\
\hline & & morillo & 20 & 21.7 \\
\hline & & solera & 15 & 16.3 \\
\hline & & tijera & 11 & 12.0 \\
\hline & & viga & 7 & 7.6 \\
\hline & & corral & 5 & 5.4 \\
\hline & & galera & 4 & 4.3 \\
\hline & & seto & 3 & 3.3 \\
\hline
\end{tabular}


Cuadro 3: Continuación.

\begin{tabular}{|c|c|c|c|c|}
\hline Categoría & Sub-categoría & Uso específico & No. Especies & Proporción \\
\hline & & regla & 2 & 2.2 \\
\hline & & tranca & 1 & 1.1 \\
\hline & & varilla & 1 & 1.1 \\
\hline \multirow[t]{4}{*}{ Religioso } & Festividades & adorno & 4 & 40.0 \\
\hline & & somé & 3 & 30.0 \\
\hline & & aromatizante & 2 & 20.0 \\
\hline & & árbol de navidad & 1 & 10.0 \\
\hline \multirow[t]{2}{*}{ Forrajera } & Forrajera & ganado bovino & 18 & 72.0 \\
\hline & & fauna silvestre & 7 & 28.0 \\
\hline
\end{tabular}

Leucaena collinsii (VUct=0.55) y Heliocarpus terebinthinaceus (VUct $=0.38$ ), fueron las especies más importantes de esta categoría.

Entre las especies de uso medicinal más significativas se encuentran Bursera simaruba (VUmd=0.46), usada principalmente en el tratamiento de enfermedades del sistema gastroinstestinal (diarrea, dolor de estómago, hemorroides e inflamación de la vesícula) y sistema urinario (inflamación de la próstata y cálculos renales); Eysenhardtia adenostylis (VUmd=0.40), usado también en desórdenes digestivos y gástricos como la diarrea y gastritis, así como de enfermedades urinarias antes mencionadas; Heliocarpus terebinthinaceus (VUmd=0.29), útil para limpiar hígado y sangre, atenuar la anemia, cálculos renales y diabetes.

Las especies de uso maderable más destacables fueron Cedrela odorata L. (VUma=1), Swietenia humilis Zucc. (VUma=0.95), Tabebuia rosea (Bertol.) Bertero ex A. DC. (VUma=0.45), Gliricidia sepium (Jacq.) Kunth (VUma $=0.37)$ y Cordia alliodora (Ruiz \& Pav.) Oken $(\mathrm{VUma}=0.3)$, las cuales fueron frecuentemente empleadas en la fabricación de muebles (mesas, sillas y camas) y partes de carreta (eje, timón y yugo). En la categoría de ornamental, las especies más valoradas fueron Sideroxylum capiri (A. DC.) Pittier y Tecoma stans, con VUor $=0.10$ y VUor $=0.18$, respectivamente. En la categoría de alimentos, Persea americana Mill. (VUa=1.07),
Byrsonima crassifolia (L.) Kunth (VUa=0.69), Annona purpurea Moc. \& Sessé ex Dunal (VUa=0.53) y Leucaena collinsii $(\mathrm{VUa}=0.5)$ fueron las especies que registraron el valor de uso más alto, especies generalmente apreciadas por el sabor de sus frutos.

Las especies de uso religioso más valiosas fueron Sideroxylum capiri (VUr=1) y Pistacia mexicana Kunth (VUr=0.4), usadas para elaborar ofrendas (enrame o somé) de los altares de santos en las festividades religiosas; Plumeria rubra L. (VUr=0.5) se empleó en la fabricación de adornos para las iglesias, mientras que Bursera excelsa (Kunth) Engl. (VUr=0.3) para sahumar con olor a incienso durante los rezos y festividades tradicionales. De los árboles usados como forraje, Acacia pennatula (VUfr=1.06) fue la especie con el valor de uso más alto. Finalmente, Bursera simaruba $(\mathrm{VUcv}=0.95)$ y $B$. excelsa $(\mathrm{VUcv}=0.35)$ fueron las especies más valoradas con la finalidad de cerco vivo.

\section{Relación entre el valor de uso y la importancia ecológica}

El valor de uso por especie no se correlacionó con el IVI, densidad, frecuencia y dominancia, denotando que las especies dominantes de la comunidad arbórea del bosque secundario no son las más valoradas por las comunidades locales. En cambio, se determinó la asociación entre el va- 
lor de uso con la dominancia relativa $\left(\mathrm{r}_{\mathrm{s}}=0.30, \mathrm{P}=0.04\right) \mathrm{de}$ las especies presentes en el sitio con 19 años de abandono. Por último, a nivel de las categorías de uso únicamente se encontró relación entre el valor de uso ornamental con la densidad de las especies $\left(r_{\mathrm{s}}=0.41, \mathrm{P}=0.03\right)$.

\section{Discusión}

\section{Riqueza etnoflorística y usos}

La riqueza de las especies arbóreas útiles registradas en este estudio representó $12 \%$ de la diversidad florística referida para los bosques secos de México por Trejo y Dirzo (2002). En términos absolutos, el número de especies encontradas en este estudio fue más alto comparado con estudios previos realizados en la región Centro de Chiapas (79) (Isidro, 1997), (99) (Isidro et al., 2006), (68) (PérezMuñoz, 2017) y aproximada a lo reportado en regiones de alto valor para la conservación del BTC en el país, como la cuenca del Río Balsas (112) (Maldonado et al., 2013) (51) (Beltrán-Rodríguez et al., 2014) (97) (ZepedaGómez et al., 2017). Aunque la curva de acumulación de las especies mencionadas por los informantes mostró que todavía es posible registrar más, el alto porcentaje de especies útiles obtenido (más de $63 \%$ del inventario de campo) es destacable, considerando el elevado grado de deterioro que posee el BTC de esta región (Ceballos et al., 2010) y la presión que genera el cambio de uso de suelo debido a la urbanización (Espinosa-Jiménez et al., 2014).

Las familias más importantes por el mayor número de especies útiles en la zona de estudio, en general, pertenecen a las más destacables de la flora del BTC (Trejo y Dirzo, 2002; Rzedowski y Calderón de Rzedowski, 2013). La predominancia de fabáceas no sorprende, ya que esta es la familia más diversa a nivel arbóreo del BTC (Sousa et al., 2001). Además, este resultado puede atribuirse al hecho de que la Depresión Central de Chiapas, a la cual pertenece al área de estudio, ha sido reconocida como una importante área de concentración de leguminosas del país (Sousa, 2010) y porque la mayor parte de las especies de esta familia poseen atributos útiles al ser humano (Hastings, 1990; Camargo-Ricalde et al., 2001).
La alta diversidad de especies útiles y el amplio número de categorías de uso muestran la estrecha relación que han tenido y tienen las comunidades campesinas con el BTC (Camou-Guerrero et al., 2008). Particularmente las categorías combustible, poste y construcción rural destacaron al concentrar un importante número de especies destinadas a satisfacer las necesidades básicas de la población. Este resultado es coincidente con las evidencias encontradas en otros estudios efectuados a lo largo del país con grupos indígenas y mestizos que por tradición han aprovechado el BTC (Sánchez-Velásquez et al., 2002; Couttolenc-Brenis et al., 2005; Moreno-Casasola y Paradowska, 2009; Martínez-Pérez et al., 2012; Suárez et al., 2012). De acuerdo con las opiniones de los entrevistados, habitantes de los asentamientos irregulares colindantes a las comunidades de estudio son los que extraen actualmente leña de forma ilícita del bosque secundario, y los informantes la obtienen de la parcela o la compran con la finalidad de preparar alimentos de gran demanda calórica (p. ej. tamales, pozol y frijoles). De igual manera los entrevistados indicaron que las especies destinadas a la construcción de la vivienda rural actualmente solo se ocupan en la estructura de galeras dentro de la casa habitación o la parcela agrícola. Este es un patrón recurrente en los núcleos agrarios de zonas urbanas y periurbanas de México, atribuido a las transformaciones económicasproductivas por el crecimiento urbano y al efecto de las normas ambientales (Salazar, 2000; Calderón-Cisneros y Soto-Pinto, 2014).

Por otra parte, el elevado número de especies destinadas al uso de poste para el cercado de parcelas y potreros está relacionado con cambios en la dinámica productiva de la región. Durante las entrevistas, los informantes expresaron que después de la entrada del Programa de Certificación de Derechos Ejidales y Titulación de Solare (PROCEDE), los terrenos agrícolas pasaron de ser de uso común a individual, obligando a cada campesino a cercar su terreno y regular la presencia de los árboles que por competencia pudieran afectar la producción de sus cultivos. No obstante, existe una tendencia por mezclar postes con cercos vivos en la delimitación de las unidades 
productivas, pues al igual que sucede en otras regiones de México y Centroamérica, los altos costos ( $\$ 10 /$ pieza de poste) y su poca durabilidad (3-5 años) obligan al campesino a buscar opciones más eficientes (Avendaño y Acosta, 2000; Harvey et al., 2003).

El uso medicinal es considerado la principal forma de aprovechamiento que los grupos indígenas y mestizos efectúan del BTC (Bye et al., 2002). El menor grado de aprecio a este grupo de plantas en las localidades de estudio puede estar relacionado con aspectos de muestreo, ya que en este trabajo no se evaluaron las herbáceas, donde se han registrado el mayor número de especies medicinales del BTC (Isidro et al., 2006; Maldonado et al., 2013; Zepeda-Gómez et al., 2017). No obstante, el uso medicinal de las especies arbóreas es un reflejo del conocimiento tradicional que aun resguardan las comunidades (Monroy y Ayala, 2003) y un recurso valioso de apoyo a la atención primaria de enfermedades frecuentemente asociadas a regiones con pobreza y marginalidad social.

Los informantes indicaron que el empleo de especies arbóreas para elaborar muebles y herramientas es un uso que va reduciéndose entre la población. El caso del uso de madera para fabricar carretas fue gradualmente sustituido por materiales más resistentes como el hierro, hasta que su uso fue prohibido como medio de transporte en la década de los años ochenta, como medida de seguridad ante el establecimiento de las vías rápidas de comunicación intermunicipales de la zona de estudio (Fig. 1). Por lo tanto, la elaboración de herramientas de trabajo fue el uso más frecuentemente mencionado por parte de los campesinos. A diferencia de lo reportado en otras regiones del país donde este uso ha sido poco reconocido (Beltrán-Rodríguez et al., 2014), su importancia mostró el corpus del conocimiento que tienen los campesinos al emplear especies en la generación de herramientas que contribuyen a sostener el sistema de cultivo tradicional (González, 2003). En cuanto a la fabricación de muebles, también es una práctica que va en desuso, esto en parte se debe a que las poblaciones silvestres de las especies frecuentemente mencionadas para este propósito, como son: Cedrela odorata, Swietenia humilis, Tabebuia rosea y Cordia alliodora, son escasas, lo cual puede observarse en los bajos valores de densidad que registraron estas especies durante el inventario florístico (Apéndice).

Las especies con valor alimenticio, ornamental, forrajero y religioso, además de ser referidas a la vegetación secundaria, son en su mayoría toleradas y cultivadas en los sistemas de producción tradicional y huertos caseros, lo cual sugiere la influencia de estos ambientes en el mantenimiento del conocimiento local y el uso actual de los recursos florísticos (Jiménez-Ferrer et al., 2008; MorenoCalles et al., 2013; Beltrán-Rodríguez et al., 2014). En particular, para el área de estudio, Isidro (1997) reportó el valor del huerto familiar en el aporte de especies vegetales destinadas a los usos ornamental, comestible y religioso en varias comunidades rurales de Tuxtla Gutiérrez. Además, Ramírez-Marcial et al. (2012) mostraron la importancia del bosque secundario asociado al sistema silvopastoril tradicional de la Depresión Central de Chiapas, al permitir la regeneración natural de una cantidad significativa de especies típicas del BTC.

\section{Relación del valor de uso e importancia ecológica}

En concordancia con las investigaciones realizadas en regiones con marcada estacionalidad climática de América tropical (Albuquerque y Lucena, 2005; Lucena et al., 2007, 2014), en el presente estudio no se encontró relación entre el valor de uso global de las especies arbóreas, basado en el consenso de los informantes con ninguno de los parámetros fitosociológicos evaluados. De acuerdo con Maldonado et al. (2013), la relación entre el valor de uso con los parámetros ecológicos se ve afectada por la alta diversidad $\beta$ y la estructura del bosque. Este segundo aspecto es particularmente crítico en la región (Ceballos et al., 2010) y en la reserva, pues la estructura del bosque secundario ha sido fuertemente impactada por la explotación de las especies de valor económico y cultural (Vargas et al., 2000; Medina et al. 2005) y, en general, por las actividades antropogénicas que provocan la dominancia ecológica de pocas especies arbóreas (Sánchez, 2014). Por lo tanto, fue la disminución de las poblaciones silvestres de las especies 
con mayor valor de uso, lo que contribuyó a no encontrar una relación entre éste y la importancia ecológica. Entonces, es probable que el valor de uso de las especies esté más relacionado con aspectos culturales y sociodemográficos (Albuquerque y Lucena, 2005; Blancas et al., 2013). Por ejemplo, Acacia pennatula fue la especie de mayor valor de uso y preferencia en el manejo del sistema silvopastoril tradicional del centro de Chiapas (Escobar-Ocampo et al., 2009; Ramírez-Marcial et al., 2012).

Es necesario tomar con cautela la correlación entre el valor de uso asociado a la importancia ecológica exhibida por el bosque de 19 años de abandono, pues estadísticamente registró un bajo nivel de asociación $\left(\mathrm{R}_{2}=0.30\right)$. En todo caso, esta débil relación es un indicio de que el valor de uso de las especies está más relacionado con la dominancia (área basal), que con la abundancia de las especies arbóreas (Lawrence et al., 2005), tal y como se ha reportado para otros bosques secos degradados del trópico americano (Ribeiro et al., 2014; Silva et al., 2014).

A pesar de que en otros estudios se han registrado relaciones significativas entre el valor de uso a nivel de las categorías construcción, alimentos y medicinales con los parámetros fitosociológicos (Lucena et al., 2007, 2012; Maldonado et al., 2013; Silva et al., 2014), en este estudio únicamente la densidad de las especies fue el parámetro asociado al mayor valor de uso en la categoría ornamental. Esta relación se explica porque las especies destinadas a la ornamentación no demandan características similares a aquellas con fines de explotación forestal (Albuquerque et al., 2015).

Las comunidades rurales del trópico poseen un importante conocimiento tradicional sobre los recursos que utilizan, clave para fomentar su participación en el diseño de estrategias para la conservación, manejo y restauración de los bosques tropicales (Uprety et al., 2012). De ahí que diversos autores hayan propuesto el valor de uso como criterio para la selección de especies arbóreas con fines de conservación, reforestación y plantaciones forestales (Suárez et al., 2012; Meli et al., 2014). Debido a que no se encontró relación entre la importancia ecológica de las especies del bosque secundario con el valor de uso, y a que las especies con mayor valor de uso registraron poblaciones silvestres reducidas, consideramos que el valor de uso puede apoyar en la identificación de especies prioritarias para la conservación y restauración del BTC en la reserva. Por esta razón, recomendamos utilizar las especies con el mayor valor de uso (Acacia pennatula y Eysenhardtia adenostylis), y otras de valor de uso intermedio (Alvaradoa amorphoides, Bursera excelsa, $B$. simaruba, Cordia alliodora, Fraxinus purpusii Brandegee, Gliricidia sepium (Jacq.) Kunth y Lysiloma acapulcense), para su utilización en programas de reforestación $\mathrm{y}$ agroforestería en apoyo a las actividades productivas de la región. La mayoría de estas especies ya han sido reconocidas como importantes para conservar y restaurar los bosques tropicales de Mesoamérica (Vázquez-Yanes et al., 1999; Cordero et al., 2003; UICN, 2015).

\section{CONCLUSIONES}

El alto porcentaje de especies con valor utilitario corrobora la importancia que tiene el BTC secundario en la provisión de especies vegetales a los habitantes de las comunidades periurbanas de la Reserva Villa Allende, siendo la familia Fabaceae la que presentó mayor número de especies. Se destaca la alta presencia de aquellas catalogadas como multipropósito y la frecuencia de uso de éstas en las categorías de combustible, construcción rural y postes, asociados a los sistemas agrícolas. Las especies Eysenhardtia adenostylis y Acacia pennatula registraron los valores de uso más alto, catalogadas como especies multipropósito que satisfacen las necesidades básicas de las familias campesinas.

La hipótesis inicialmente planteada en este estudio no se cumplió debido a que encontramos una nula o débil relación entre el valor de uso por especie y los parámetros fitosociológicos de dominancia, densidad, frecuencia e índice de importancia ecológica, lo que demuestra que el valor de uso no está determinado por la importancia ecológica de la especie. Asimismo, la relación valor de uso e índice de importancia ecológica puede estar afectada por el estado de perturbación de la estructura del bosque secundario. Es necesario que las especies identificadas con 
mayor valor de uso y, en particular, las de baja dominancia ecológica se investiguen más a detalle para determinar si tienen alguna presión debido a su utilización pues se carece de información sobre las prácticas de manejo tradicional y las condiciones ambientales sobre el espacio y tiempo en que sucede su aprovechamiento. Los planes y programas para la conservación, manejo y restauración del BTC en el área de estudio deben además de considerar los aspectos ecológicos de las especies, el conocimiento tradicional que poseen las comunidades locales y las dinámicas que los grupos mestizos enfrentan ante el proceso de urbanización.

\section{CONTRIBUCIÓN DE AUTORES}

LARL, MSSC y MCGR concibieron y diseñaron el estudio. MCGR realizó los análisis, contribuyó con la adquisición de datos y su interpretación, y escribió el documento con la ayuda de LARL y MSSC. Todos los autores contribuyeron a la discusión, revisión y aprobación del manuscrito final.

\section{FINANCIAMIENTO}

Este estudio fue financiado por el Gobierno del Estado de Chiapas a través de la Secretaria de Medio Ambiente e Historia Natural (proyecto 042151S09201E10A003000). El apoyo se le otorgó al autor MCGR para solventar el trabajo de campo.

\section{AgRADECIMIENTOS}

Se agradece a las autoridades locales y habitantes de las comunidades por participar en este estudio. A la Secretaria de Medio Ambiente e Historia Natural por el apoyo brindado en la gestión de permisos de colecta, logística y acceso al herbario CHIP. A los curadores Oscar Farrera Sarmiento (Herbario CHIP), Miguel Ángel Pérez Farrera y Josefa Anahí Espinosa Jiménez (Herbario Eizi Matuda) por su apoyo en la identificación taxonómica de las colectas botánicas. A los estudiantes Daniel Pérez Muñoz, Diana Yanet Sánchez Molina, Dalia Elizabeth Sántiz Gómez y Diego Velázquez Suárez, por su apoyo en la adquisición de los datos de campo y entrevistas.

\section{LITERATURA CITADA}

Albuquerque, U. P. y R. P. F. Lucena. 2005. Can apparency affect the use of plants by local people in tropical forest? Interciencia 8(30): 506-511.

Albuquerque, U. P., G. S. Taboada, M. R. Alves, J. Gomes de Melo, P. M. Muniz, A. N. Borba y W. J. Ferreira. 2015. The influence of the environment on natural resource use: evidence of apparency. In: Albuquerque, U. P., P. M. Muniz and A. Casas (eds.). Evolutionary Ethnobiology. Springer International Publishing. Basel, Switzerland. Pp. 131-147. DOI: https://dx.doi.org/10.1007/978-3-31919917-7_10

APG IV. 2016. An update of the Angiosperm Phylogeny Group classification for the orders and families of flowering plants: APG IV. Botanical Journal of the Linnean Society 181(1): 1-20. DOI: https://dx.doi.org/10.1111/boj.12385

Avendaño, R. S. y R. I. Acosta. 2000. Plantas utilizadas como cercas vivas en el estado de Veracruz. Madera y Bosques 6(1): 55-71. DOI: https://doi.org/6.10.21829/ myb.2000.611342

Balvanera, P. y M. Maass. 2010. Los servicios ecosistémicos que proveen las selvas secas. In: Ceballos, G., L. Martínez, A. García, E. Espinoza, J. B. Creel y R. Dirzo (eds.). Diversidad, amenazas y áreas prioritarias para la conservación de las selvas secas del Pacífico de México. Comisión Nacional para el Conocimiento y Uso de la Biodiversidad-Fondo de Cultura Económica. México, D.F., México. Pp. 251-270.

Balvanera, P., A. Castillo y M. Martínez-Harms. 2011. Ecosystem Services in seasonally dry tropical forests. In: Dirzo, R., H. S. Young, H. A. Mooney y G. Ceballos (eds.). Seasonally dry tropical forests. Island Press. Washington, D.C., USA. Pp. 259-277. DOI: https://doi. org/10.5822/978-1-61091-021-7_15

Beltrán-Rodríguez, L., A. Ortiz-Sánchez, N. A. Mariano, B. Maldonado-Almanza y V. Reyes-García. 2014. Factors affecting ethnobotanical knowledge in a mestizo community of the Sierra de Huautla Biosphere Reserve, Mexico. Journal of Ethnobiology and Ethnomedicine 10(1): 14. DOI: https://doi.org/10.1186/1746-4269-1014 
Berkes, F., J. Colding y C. Folke. 2000. Rediscovery of traditional ecological knowledge as adaptive management. Ecological applications 10(5): 1251-1262. DOI: https:// doi.org/10.1890/1051-0761(2000)010[1251:ROTEKA]2 $.0 . \mathrm{CO} ; 2$

Blancas, J., A. Casas, D. Pérez-Salicrup, J. Caballero y E. Vega. 2013. Ecological and socio-cultural factors influencing plant management in Náhuatl communities of the Tehuacán Valley, Mexico. Journal of Ethnobiology and Ethnomedicine 9(1): 39. DOI: https://doi. org/10.1186/1746-4269-9-39

Bye, R. 1995. Ethnobotany of the Mexican dry tropical forests. In: Bullock, S. H., H. A. Mooney y E. Medina (eds.). Seasonally dry tropical forests. Cambridge University Press. Cambridge, UK. Pp. 423-438.

Bye, R., L. Cervantes y B. Rendón. 2002. Etnobotánica en la región de Chamela, Jalisco, México. In: Noguera, F., J. Vega, A. García y M. Quesada (eds.). Historia natural de Chamela. Instituto de Biología, Universidad Nacional Autónoma de México. México D.F., México. Pp. 545-559.

Calderón-Cisneros, A. y L. Soto-Pinto. 2014. Transformaciones agrícolas en el contexto periurbano de la ciudad de San Cristóbal de Las Casas, Chiapas. LiminaR Estudios Sociales y Humanísticos 12(1): 125-143. DOI: https://doi. org/10.29043/liminar.v12i1.329

Camargo-Ricalde, S. L., R. Grether, A. Martínez-Bernal, V. García-García y S. Barrios. 2001. Especies útiles del género Mimosa (Fabaceae-Mimosoideae) en México. Boletín de la Sociedad Botánica de México 68: 33-44. DOI: https://dx.doi.org/10.17129/botsci.1634

Camou-Guerrero, A., V. Reyes-García, M. Martínez-Ramos y A. Casas. 2008. Knowledge and use value of plant species in a Rarámuri Community: a gender perspective for conservation. Human Ecology 36: 259-272. DOI: https:// dx.doi.org/10.1007/s10745-007-9152-3

Ceballos, G., L. Martínez, A. García, E. Espinoza, J. B. Creel y R. Dirzo. 2010. Diversidad, amenazas y áreas prioritarias para la conservación de las selvas secas del Pacífico de México. Comisión Nacional para el Conocimiento y Uso de la Biodiversidad-Fondo de Cultura Económica. México, D.F., México. 594 pp.
Challenger, A. y J. Soberón. 2008. Los ecosistemas terrestres. In: Soberón, J., G. Halffter y J. Llorente-Bousquets (eds.). Capital natural de México, Vol. I: Conocimiento actual de la biodiversidad. Comisión Nacional para el Conocimiento y Uso de la Biodiversidad. México, D.F., México. Pp. 87-108.

Cordero, J., D. H. Boshier, CATIE y OFI. 2003. Árboles de Centroamérica: un manual para extensionistas. Oxford Forestry Institute-Centro Agronómico Tropical de Investigación y Enseñanza. Oxford, UK. 1079 pp.

Couttolenc-Brenis, E., J. A. Cruz-Rodríguez, E. Cedillo Portugal y M. A. Musálem. 2005. Uso local y potencial de las especies arbóreas en Camarón de Tejeda, Veracruz. Revista Chapingo Serie Ciencias Forestales y del Ambiente 11(1): 45-50.

Cruz-Burguete, J. L. y P. E. Almazán-Esquivel. 2008. Los Zoques de Tuxtla y la disputa por las virgencitas de Copoya, en el valle central de Chiapas. Ra Ximhai 4(2): 21-47.

Díaz, S., J. Fargione, F. C. Stuart y D. Tilman. 2006. Biodiversity loss threatens human well-being. PLoS Biology 4(8): e277. DOI: https://doi.org/10.1371/ journal.pbio.0040277

Escobar-Ocampo, C., J. C. Niños, N. Ramírez-Marcial y C. P. Yépez. 2009. Diagnóstico participativo del uso, demanda y abastecimiento de leña en una comunidad zoque del centro de Chiapas, México. Ra Ximhai 5(2): 201-223.

Espinosa-Jiménez, J. A., A. López-Cruz, M. A. Pérez-Farrera y S. López. 2014. Inventario florístico de la cañada la Chacona-Juan Crispín y zonas adyacentes, Depresión Central de Chiapas, México. Botanical Sciences 92(2): 205-241. DOI: https://dx.doi.org/10.17129/botsci.30

Feeny, P. 1976. Plant apparency and chemical defense. In: Wallace, J. W. y R. L. Mansell (eds.). Biochemical interaction between plants and insects. Recent Advances in Phytochemistry, vol. 10. Springer. Boston, USA. Pp. 1-40. DOI: https://doi.org/10.1007/978-1-4684-2646-5_1

Garibaldi, A. y N. Turner. 2004. Cultural keystone species: implications for ecological conservation and restoration. Ecology and Society 9(3): 1. DOI: https://doi.org/10.5751/ es-00669-090301 
González, J. A. 2003. Cultura y agricultura: transformaciones en el agromexicano. México. Universidad Iberoamericana. México, D.F., México. 343 pp.

Goodman, L. A. 1961. Snowball sampling. The Annals of Mathematical Statistics 32(1): 148-170. DOI: https://doi. org/10.1214/aoms/1177705148

Harvey, C. A., J. V. Villanueva, M. Chacón, D. Muñoz y M. López. 2003. Contribución de las cercas vivas a la productividad e integridad ecológica de los paisajes agrícolas en América Central. Agroforestería en las Américas 10: 30-39.

Hastings, R. B. 1990. Medicinal legumes of Mexico: Fabaceae, Papilionoideae, part one. Economic Botany 44(3): 336348. DOI: https://doi.org/10.1007/BF03183915

Hickey, G. M., M. Pouliot, C. Smith-Hall, S. Wunder y M. R. Nielsen. 2016. Quantifying the economic contribution of wild food harvests to rural livelihoods: a globalcomparative analysis. Food Policy 62: 122-132. DOI: https://doi.org/10.1016/j.foodpol.2016.06.001

Holz, S. y G. Placci. 2008. El desafío de la restauración de bosques en paisajes poblados: un enfoque multidisciplinar en Misiones, Argentina. In: GonzálezEspinosa, M., J. M. Rey-Benayas y N. Ramírez-Marcial (eds.). Restauración de bosques en América Latina. Fundación Internacional para la Restauración de Ecosistemas (FIRE) y Editorial Mundi-Prensa. México, D.F., México. Pp. 163-179.

Huerta García, M. A., R. Escalante López, A. Méndez Barrera, P. Sánchez Montero, K. Leal Aguilar, A. Rodríguez Jiménez, M. M. Torres Álvarez y B. Gálvez Cruz. 2016. Paisajes transformados y el cambio climático: el caso de las áreas naturales protegidas de la Ecoregión "Selva Zoque", Chiapas. In: Escuinca Cano, F. y M. C. Gordillo Ruiz (coord.). Ecoregión Zoque: retos y oportunidades ante el cambio climático. Gobierno del Estado de Chiapas, Secretaria de Medio Ambiente e Historia Natural. Tuxtla Gutiérrez, México. Pp. 144-164.

INEGI. 1999. Modelo de elevación digital 1:50,000 Tuxtla Gutiérrez E15C59 y E15C69. Instituto Nacional de Estadística, Geografía e Informática. Aguascalientes, México.
INEGI. 2000. Carta edafológica 1:250,000 Tuxtla Gutiérrez E15-11. Instituto Nacional de Estadística, Geografía e Informática. Aguascalientes, México.

INEGI. 2003. Conjunto de datos vectoriales de la carta de vegetación primaria 1:1,000,000. Instituto Nacional de Estadística, Geografía e Informática. Aguascalientes, México.

INEGI. 2010. XIII Censo general de población y vivienda. Instituto Nacional de Estadística, Geografía e Informática. Aguascalientes, México. http://www.inegi.org.mx/ est/contenidos/proyectos/ccpv/cpv2010/ (consultado septiembre de 2017).

INI. 1994. Biblioteca de la Medicina Tradicional Mexicana. Primera edición digital. Universidad Nacional Autónoma de México. Cd. Mx., México. http://www. medicinatradicionalmexicana.unam. $\mathrm{mx} / \mathrm{index} . \mathrm{php}$ (consultado enero de 2018).

IPNI. 2012. The International Plant Names Index. http://www. ipni.org. (consultado noviembre de 2017).

Isidro, M. V. 1997. Etnobotánica de los Zoques de Tuxtla Gutiérrez, Chiapas. Instituto de Historia Natural. Tuxtla Gutiérrez, México. 125 pp.

Isidro, M. V. y O. Farrera S. 1999. Uso tradicional de las plantas por los Zoques de Chiapas. Fin de Siglo 2(1): 7-9.

Isidro, M. V., N. Moreno y O. Farrera S. 2006. Planta útiles de los zoques del centro de Chiapas. In: Aramoni, D. C., T. W. Lee. y M. G. Lisbona (eds.). Presencia Zoque: una aproximación interdisciplinaria. Universidad de Ciencias y Artes de Chiapas, Consejo de Ciencia y Tecnología del Estado de Chiapas-Universidad Autónoma de ChiapasUniversidad Nacional de México. México, D.F., México. Pp. 369-386.

Janzen, H. D. 1988. Management of habitat fragments in a tropical dry forest: growth. Annals of the Missouri Botanical Garden 75(1): 105-116. DOI: https://doi. org $/ 10.2307 / 2399468$

Jiménez-Ferrer, G., R. Velasco-Pérez, M. U. Gómez y L. SotoPinto. 2008. Ganadería y conocimiento local de árboles y arbustos forrajeros de la Selva Lacandona, Chiapas, México. Zootecnia Tropical 26(3): 333-337.

Kalacska, M., G. A. Sánchez-Azofeita, J. C. Calvo-Alvarado, M. Quesada, B. Rivardy y D. H. Janzen. 2004. Species 
composition, similarity and diversity in three successional stages of a seasonally dry tropical forest. Forest Ecology and Management 200(1-3): 227-247. DOI: https:/doi. org/10.1016/j.foreco.2004.07.001

Lawrence, A., O. L. Phillips, M. Reategui, S. Lopes, D. Rose y A. J. Farfan. 2005. Local values for harvested forest plants in Madre de Dios, Peru: towards a more contextualized interpretation of quantitative ethnobotanical data. Biodiversity and Conservation 14(1): 45-79. DOI: https:// doi.org/10.1007/s10531-005-4050-8

Lisbona, M. G. 2006. Olvidados del neozapatismo: los zoques chiapanecos. Estudios sociológicos 24(71): 305-330.

López, E. J. G. 2006. Estimación de tormentas y avenidas para el diseño de las obras de protección del Río Sabinal. Tesis de maestría. Universidad Autónoma de Chiapas. Tuxtla Gutiérrez, Chiapas, México. 70 pp.

Lucena, R. F. P., U. P. Albuquerque y E. L. Araújo. 2007. Does the use-value of woody plants of the Caatinga (Northeastern Brazil) explain their local availability? Economic Botany 61(4): 347-361. DOI: https://doi.org/10.1663/00130001(2007)61[347:DTLAOW]2.0.CO;2

Lucena, R. F. P., P. M. Medeiros, E. L. Araújo, A. A. Chaves y U. P. Albuquerque. 2012. The ecological apparency hypothesis and the importance of useful plants in rural communities from Northeastern Brazil: An assessment based on use value. Journal of Environmental Management 96(1): 106-115. DOI: https://doi.org/10.1016/j.jenvman.2011.09.001

Lucena, R. F. P., R. F. Sousa, N. M. Guerra, J. E. S. Ribeiro, A. P. Leite, D. B. O. Abreu, T. K. N. Carvalho, D. M. B. M. Trovão, C. A. B. Alves, R. R. N. Alves, P. F. Borges, L. A. Andrade, J. S. Souto, S. P. Sousa y E. N. Nunes. 2014. The ecological apparency hypothesis and dry tropical forests: an ethnobotanical assessment. Etnoecológica 10(9): 1-17.

Maffi, L. 2005. Linguistic, cultural and biological diversity. Annual ReviewAnthropology34(1):599-617.DOI:https:// doi.org/10.1146/annurev.anthro.34.081804.120437

Maldonado, B., J. Caballero, A. Delgado-Salinas y R. Lira. 2013. Relationship between use value and ecological importance of floristic resources of seasonally dry tropical forest in the Balsas River Basin, Mexico. Economic
Botany 67(1): 17-29. DOI: https://doi.org/10.1007/ s12231-013-9222-y

Martínez-Pérez, A., P. A. López, A. Gil-Muñoz y A. CuevasSánchez. 2012. Plantas silvestres útiles y prioritarias identificadas en La Mixteca Poblana, México. Acta Botanica Mexicana 98: 73-98. DOI: https://doi. org/10.21829/abm98.2012.1141

Medina, S. L., G. M. Anaya, H. V. Volke y C. S. Ortiz. 2005. Formulación de un plan de desarrollo agropecuario y forestal para una comunidad ejidal del municipio de San Fernando, Chiapas, México. Mundo Agrario 5: 13741405.

Meli, P., M. Martínez-Ramos, J. Rey-Benayas y J. Carabias. 2014. Combining ecological, social and technical criteria to select species for restoration. Applied Vegetation Science 17(4): 744-753. DOI: https://doi.org/10.1111/ avsc. 12096

Miles, L., A. C. Newton, R. S. DeFries, C. Ravilious, I. May, S. Blyth, V. Kapos y J. E. Gordon. 2006. A global overview of the conservation status of tropical dry forests. Journal of Biogeography 33(3): 491-505. DOI: https://doi. org/10.1111/j.1365-2699.2005.01424.x

Monroy, R. e I. Ayala. 2003. Importancia del conocimiento etnobotánico frente al proceso de urbanización. Etnobiología 3: 79-92.

Moreno-Calles, A., V. Toledo y A. Casas. 2013. Los sistemas agroforestales tradicionales de México: una aproximación biocultural. Botanical Sciences 91(4): 375-398. DOI: http://dx.doi.org/10.17129/botsci.419

Moreno-Casasola, P. y K. Paradowska. 2009. Especies útiles de la selva baja caducifolia en las dunas costeras del centro de Veracruz. Madera y Bosques 15(3): 21-4. DOI: https:// doi.org/10.21829/myb.2009.1531184

Mueller-Dombois, D. y H. Ellenberg. 2002. Aims and methods of vegetation ecology. Second Edition. Blackburn Press. Caldwell, USA. 547 pp.

Mulleried, F. 1951. Geología de Chiapas. Colección libros de Chiapas, Serie Básica. Gobierno del Estado de Chiapas. Tuxtla Gutiérrez, México. 180 pp.

Murphy, P. G. y A. E. Lugo. 1995. Dry forest of Central America and the Caribbean. In: Bullock, S. H., H. A. Mooney y E. 
Medina (eds.). Seasonally dry tropical forests. Cambridge University Press. Cambridge, UK. Pp. 9-34.

OPS. 1995. Clasificación estadística internacional de enfermedades y problemas relacionados con la salud. Décima revisión. Organización Panamericana de la Salud. Washington, D.C., USA. http://ais.paho.org/ classifications/Chapters/pdf/Volume2.pdf (consultado enero de 2018).

Pérez-Muñoz, D. A. 2017. Evaluación de la composición, estructura y usos de la comunidad vegetal leñosa al sur de la zona protectora foresta vedada "Villa Allende", Chiapas, México. Tesis de licenciatura. Instituto de Ciencias Biológicas, Universidad de Ciencias y Artes de Chiapas. Tuxtla Gutiérrez, Chiapas, México. 113 pp.

Phillips, O. y A. H. Gentry. 1993. The useful plants of Tambopata, Peru: I. Statistical hypothesis tests with a new quantitative technique. Economic Botany 47(1): 15-32. DOI: https://doi.org/10.1007/BF02862203

Quesada, M., G. A. Sánchez-Azofeifa, M. Álvarez-Anorve, K. E. Stoner, L. Ávila-Cabadilla, J. Calvo-Alvarado, A. Castillo, M. M. Espirito-Santo, M. Fagundes, G. W. Fernandes, J. Gamon, M. Lopezaraiza-Mikel, D. Lawrence, L. P. Cerdeira Morellato, J. S. Powers, F. S. Neves, V. Rosas-Guerrero, R. Sayago y G. Sánchez-Montoya. 2009. Succession and management of tropical dry forests in the Americas: review and new perspectives. Forest Ecology Management 258(6): 1014-1024. DOI: https://doi.org/10.1016/j. foreco.2009.06.023

Ramírez-Marcial, N., M. L. Rueda-Pérez, B. Ferguson y G. Jiménez-Ferrer. 2012. Caracterización del sistema agrosilvopastoril en la Depresión Central de Chiapas. Avances en Investigación Agropecuaria 16(2): 7-22.

R Core Team. 2013. R: A language and environment for statistical computing. R Foundation for Statistical Computing. Vienna, Austria. https://www.r-project.org/

Reyes-García, V., T. Huanca, V. Vadez, W. Leonard y D. Wilke. 2006. Cultural,practical, andeconomicvalueofwildplants: a quantitative study in the Bolivian Amazon. Economic Botany 60(1): 62-74. DOI: https://doi.org/10.1663/00130001(2006)60[62:CPAEVO]2.0.CO;2
Ribeiro, J. P. O., T. K. N. Carvalho, J. E. S. Ribeiro, R. F. Sousa, J. R. F. Lima, C. A. B. Alves, J. G. Jardim y R. F. P. Lucena. 2014. Can ecological apparency explain the use of plant species in the semi-arid depression of Northeastern Brazil? Acta Botanica Brasilica 28(3): 476-483. DOI: https://dx.doi.org/10.1590/0102-33062014abb2758

Rocha-Loredo, A. C., N. Ramírez-Marcial y M. GonzálezEspinosa. 2010. Riqueza y diversidad de árboles del bosque tropical caducifolio en la Depresión Central de Chiapas. Botanical Sciences 87: 89-103. DOI: http:// dx.doi.org/10.17129/botsci.313

Rossato, S., H. F. Leitáo-Filho y A. Begossi. 1999. Ethnobotany of Caicaras of the Atlantic Forest Coast (Brazil). Economic Botany 53(4): 387-395. DOI: https://doi.org/10.1007/ BF02866716

Rzedowski, J. 1978. La vegetación de México. Limusa, S.A., México, D.F., México. 432 pp.

Rzedowski, J. 2006. Vegetación de México. Primera edición digital. Comisión Nacional para el Conocimiento y Uso de la Biodiversidad. México, D.F., México. http:// www.biodiversidad.gob.mx/publicaciones/librosDig/ pdf/VegetacionMx_Cont.pdf (consultado junio de 2017).

Rzedowski, J. y G. Calderón de Rzedowski. 2013. Datos para la apreciación de la flora fanerogámica del bosque tropical caducifolio de México. Acta Botanica Mexicana 102: 1-23. DOI: https://doi.org/10.21829/abm102.2013.229

Salafsky, N. y E. Wollenberg. 2000. Linking livelihoods and conservation: a conceptual framework and scale for assessing the integration of human needs and biodiversity. World Development 28(8): 1421-1438. DOI: https://doi. org/10.1016/S0305-750X(00)00031-0

Salazar, C. C. 2000. La relación población-recursos en la periferia urbana: una experiencia teórica-metodológica. Estudios demográficos y urbanos 15(3): 641-664. DOI: https://dx.doi.org/10.24201/edu.v15i3.1089

Sánchez, M. D. 2014. Estructura y composición florística de la subcuenca del río Sabinal, Chiapas, México. Tesis de licenciatura. Instituto de Ciencias Biológicas, Universidad de Ciencias y Artes de Chiapas. Tuxtla Gutiérrez, Chiapas, México. 97 pp. 
Sánchez-Velásquez, L., G. Hernández-Vargas, M. M. A. Carranza, M. R. Pineda-López, G. R. Cuevas y C. F. Aragón. 2002. Estructura del bosque tropical caducifolio usado para la ganadería extensiva en el norte de la sierra de Manantlán, México: antagonismos de usos. Polibotánica 13: 25-46.

Silva, R. J. R., T. C. Nunes, J. R. Oliveira, N. G. Medeiros, N. Silva, K. P. Marques, C. A. Belarmino, S. J. Sousa, J. S. Silva, A. N. Trajano, J. L. Ribamar, R. Silva de Oliveira y R. L. Farias. 2014. Ecological apparency hypothesis and availability of useful plants: testing different use values. Ethnobotany Research and Applications 12: 415-432. DOI: https://dx.doi.org/10.17348/era.12.0.415-432

Sousa, M. S. 2010. Centro de endemismos: las leguminosas. In: Ceballos, G., L. Martínez, A. García, E. Espinoza, J. B. Creel y R. Dirzo. (eds.). Diversidad, amenazas y áreas prioritarias para la conservación de las selvas secas del Pacífico de México. Comisión Nacional para el Conocimiento y Uso de la Biodiversidad-Fondo de Cultura Económica. México, D.F., México. Pp. 77-91.

Sousa, M. S., M. Ricker y H. M. Hernández. 2001. Tree species of the family Leguminosae in Mexico. Harvard Papers in Botany 6(1): 339-365.

Suárez, A., G. Williams-Linera, C. Trejo, J. I. Valdez-Hernández, V. M. Cetina-Alcalá y H. Vibrans. 2012. Local knowledge helps select species for forest restoration in tropical dry forest of central Veracruz, Mexico. Agroforestry Systems 85(1): 35-55. DOI: https://doi.org/10.1007/s10457-0119437-9

Trejo, I. y R. Dirzo. 2002. Floristic diversity of Mexican seasonally dry tropical forests. Biodiversity and Conservation 11(11): 2063-2084. DOI: https://doi. org/10.1023/A:1020876316013

Turner, N. J. 1988. "The importance of a rose": Evaluating the cultural significance of plants in Thompson and Lillooet Interior Salish. American Anthropologist 90(2): 272-290. DOI: https://doi.org/10.1525/aa.1988.90.2.02a00020

UICN. 2015. Especies para restauración. Unión Internacional para la Conservación de la Naturaleza. http://www. especiesrestauracion-uicn.org/index.php (consultado enero de 2018).
Uprety, Y., H. Asselin, Y. Bergeron, F. Doyon y J. F. Boucher. 2012. Contribution of traditional knowledge to ecological restoration: practices and applications. Ecoscience 19(3): 225-237. DOI: https://doi.org/10.2980/19-3-3530

Vaca, A. R., J. D. Golicher, L. Cayuela, J. Hewson y M. Steininger. 2012. Evidence of incipient forest transition in Southern Mexico. PLoS ONE 7: e42309. DOI: https://doi. org/10.1371/journal.pone.0042309

Vargas, M. F., S. Escobar y R. Ángel. 2000. Áreas Naturales Protegidas de México con decretos federales. Instituto Nacional de Ecología-Secretaría de Medio Ambiente, Recursos Naturales y Pesca-Red para el Desarrollo Sostenible, A.C.-Programa de Naciones Unidas para el Desarrollo. México, D.F., México. 830 pp.

Vázquez-Yanes, C. A., I. Batis M., M. I. Alcocer S., M. Gual D. y C. Sánchez D. 1999. Árboles y arbustos nativos potencialmente valiosos para la restauración ecológica y la reforestación. Reporte técnico del proyecto J084. Comisión Nacional para el Conocimiento y Uso de la Biodiversidad-Instituto de Ecología, Universidad Nacional Autónoma de México. http://www.conabio.gob. $\mathrm{mx} /$ conocimiento/info_especies/arboles/doctos/inicio.pdf (consultado enero de 2018).

Vieira, D. L. M. y A. Scariot. 2006. Principles of natural regeneration of tropical dry forests for restoration. Restoration Ecology 14(1): 11-20. DOI: https://doi. org/10.1111/j.1526-100X.2006.00100.x

Williams, V. L., T. F. Edward y K. B. Witkowski. 2007. The use of incidence-based species richness estimators, species accumulation curves and similarity measures to appraise ethnobotanical inventories from South Africa. Biodiversity Conservation 16(9): 2495-2513. DOI: https://doi.org/10.1007/s10531-006-9026-9

Zárate, S. 1999. Ethnobotany and domestication process of Leucaena in Mexico. Journal of Ethnobiology 19(1): $1-23$.

Zepeda-Gómez, C., C. A. Burrola, L. O. White y S. C. Rodríguez. 2017. Especies leñosas útiles de la selva baja caducifolia en la Sierra de Nanchititla, México. Madera y Bosques 23(3): 101-119. DOI: https://dx.doi. org/10.21829/myb.2017.2331426 
Apéndice: Categorías de uso, valor de uso e importancia ecológica de las especies arbóreas del bosque tropical caducifolio secundario en la Reserva Forestal Villa Allende, Chiapas. VU=índice de valor de uso, IVI=índice de valor de importancia ecológica, Do=dominancia, De=densidad, $\mathrm{Fr}=$ frecuencia. Categorías de uso: $\mathrm{a}=$ alimento, $\mathrm{b}=$ construcción rural, $\mathrm{c}=$ cerco vivo, $\mathrm{d}=$ religioso, $\mathrm{e}=$ forraje, $\mathrm{f}=$ maderable, $\mathrm{g}=$ medicinal, $\mathrm{h}=\mathrm{ornamental}$, $\mathrm{i}=$ poste, $\mathrm{j}=$ combustible. Usos específicos: $0=$ adorno, $1=$ ampollas, $2=$ anemia, 3=árbol de navidad, 4=aromatizante, 5=bebida, 6=cabo de coa, $7=$ cabo de hacha, $8=$ cálculos renales, $9=$ cama, $10=$ canoa, $11=$ cercha, $12=$ cocimiento de panela, $13=$ cocimiento de ladrillo, $14=$ colgador, $15=$ colitis, $16=$ condimento, $17=$ corral, $18=$ depurativa sangre, 19=depurativa hígado, $20=$ desinflamar vesícula, $21=$ diabetes, $22=$ diarrea, $23=$ dolor de cabeza, $24=$ dolor de estómago, $25=$ dolor de hueso, 26=dolor de muela, $27=$ dolor muscular, $28=$ dulces, $29=$ eje, $30=$ escalera, $31=$ escoba, $32=$ esquinero, $33=$ estreñimiento, $34=$ fauna silvestre, $35=$ frutas, 36=galera, 37=ganado bovino, $38=$ gastritis, 39=golpes, $40=$ gripa, 41=guisado, $42=$ hemorragia, 43=hemorroides, 44=heridas, 45=horcón, 46=desinflamar la próstata, 47=desinflamar órganos, 48=juguete, 49=larguero, 50=leña, 51=mal de aire, 52=mal de espanto, 53=masa, 54=mesa, 55=montura, 56=morillo, 57=parásitos, 58=puerta, 59=rayo, 60=regla, 61=regular la menstruación, 62=reja, $63=$ sarampión, $64=$ seto, $65=$ silla, $66=$ solera, $67=$ somé, $68=$ tamales, $69=$ tijera, 70=timón, $71=$ timón de arado, 72=tos, 73=tranca, 74=úlcera, $75=$ varilla, $76=$ viga y $77=$ yugo. Los ejemplares de herbarios se depositaron en el herbario de la Secretaria de Medio Ambiente e Historia Natural (CHIP) y en el herbario Eizi Matuda (HEM), y los registros fotográficos (reg. fot.) se pueden solicitar a través del autor de correspondencia. Nombre de los colectores: $D A P M=$ Daniel Alejandro Pérez Muñoz, DYSM=Diana Yaneth Sánchez Muñoz, MCGR=Mercedes Concepción Gordillo Ruiz.

\begin{tabular}{|c|c|c|c|c|c|c|c|c|c|}
\hline Familia/especie & $\begin{array}{l}\text { Nombre } \\
\text { común }\end{array}$ & No. Colecta & Usos & $\begin{array}{c}\text { Usos } \\
\text { específicos }\end{array}$ & VU & IVI & Do & De & $\mathrm{Fr}$ \\
\hline \multicolumn{10}{|l|}{ ANACARDIACEAE } \\
\hline $\begin{array}{l}\text { Comocladia guatemalensis Donn. } \\
\text { Sm. }\end{array}$ & cinco negritos & $D A P M 191$ & $b, f, g, i, j$ & $\begin{array}{l}17,29,38,40 \\
44,45,50,66\end{array}$ & 0.35 & 0.27 & 0.17 & 0.28 & 0.36 \\
\hline Pistacia mexicana Kunth & achin & DYSM 593 & $b, f, g, i, j$ & $\begin{array}{c}21,45,50,53 \\
67,71\end{array}$ & 0.24 & - & - & - & - \\
\hline Rhus schiedeana Schltdl. & ocotillo & DYSM 593 & $\mathrm{i}, \mathrm{j}$ & 50 & 0.04 & - & - & - & - \\
\hline Spondias mombin L. & jobo & $M C G R 201$ & $\mathrm{a}, \mathrm{i}, \mathrm{j}$ & 35,50 & 0.04 & - & - & - & - \\
\hline Spondias purpurea L. & jocote de caballo & $D A P M 114$ & $\mathrm{a}, \mathrm{c}, \mathrm{e}, \mathrm{j}$ & $12,37,50$ & 0.08 & 0.08 & 0.03 & 0.04 & 0.18 \\
\hline \multicolumn{10}{|l|}{ ANNONACEAE } \\
\hline $\begin{array}{l}\text { Annona macroprophyllata Donn. } \\
\text { Sm. }\end{array}$ & papausa & Reg. fot. & $\mathrm{a}, \mathrm{g}, \mathrm{i}, \mathrm{j}$ & $35,40,47,50$ & 0.13 & - & - & - & - \\
\hline Annona muricata L. & guanábana & Reg. fot. & $\mathrm{a}, \mathrm{g}$ & 35 & 0.10 & - & - & - & - \\
\hline $\begin{array}{l}\text { Annona purpurea Moc. \& Sessé ex } \\
\text { Dunal }\end{array}$ & chincuya & MCGR 170 & $\mathrm{a}, \mathrm{i}, \mathrm{j}$ & 35,50 & 1.86 & 0.39 & 0.15 & 0.32 & 0.71 \\
\hline Annona reticulata $\mathrm{L}$. & $\begin{array}{l}\text { anon, annona } \\
\text { amarilla }\end{array}$ & Reg. fot. & $\mathrm{a}, \mathrm{g}, \mathrm{j}$ & $\begin{array}{l}22,23,25,27 \\
35,40,42,50\end{array}$ & 0.21 & - & - & - & - \\
\hline \multicolumn{10}{|l|}{ APOCYNACEAE } \\
\hline Cascabela ovata (Cav.) Lippold & manzanito & MCGR 120 & $\mathrm{~b}$ & 45 & 0.01 & 1.13 & 1.26 & 0.87 & 1.25 \\
\hline Plumeria rubra $\mathrm{L}$. & $\begin{array}{l}\text { flor de mayo, } \\
\text { chimpipi }\end{array}$ & MCGR 35 & $\mathrm{~d}, \mathrm{~g}, \mathrm{~h}$ & 0 & 0.07 & 0.22 & 0.21 & 0.08 & 0.36 \\
\hline \multicolumn{10}{|l|}{ ARALIACEAE } \\
\hline $\begin{array}{l}\text { Dendropanax arboreus (L.) Decne. } \\
\text { \& Planch. } \\
\text { ASPARAGACEAE }\end{array}$ & palo coleto & DYSM 570 & $\mathrm{~b}$ & - & 0.01 & - & - & - & - \\
\hline $\begin{array}{l}\text { Beaucarnea goldmanii Rose } \\
\text { BIGNONIACEAE }\end{array}$ & pata de elefante & Reg. fot. & $\mathrm{h}$ & - & 0.01 & - & - & - & - \\
\hline $\begin{array}{l}\text { Parmentiera aculeata (Kunth) } \\
\text { Seem. }\end{array}$ & cuajilote & MCGR 203 & $\mathrm{e}$ & 37 & 0.01 & - & - & - & - \\
\hline Tabebuia rosea (Bertol.) DC. & matilisguate & MCGR 189 & $\mathrm{~b}, \mathrm{c}, \mathrm{f}, \mathrm{g}, \mathrm{h}, \mathrm{j}$ & $\begin{array}{l}9,47,49,50 \\
54,58,65,66 \\
76,77\end{array}$ & 0.35 & 0.10 & 0.08 & 0.04 & 0.18 \\
\hline
\end{tabular}


Apéndice: Continuación.

\begin{tabular}{|c|c|c|c|c|c|c|c|c|c|}
\hline Familia/especie & $\begin{array}{l}\text { Nombre } \\
\text { común }\end{array}$ & No. Colecta & Usos & $\begin{array}{c}\text { Usos } \\
\text { específicos }\end{array}$ & $\mathrm{VU}$ & IVI & Do & $\mathrm{De}$ & $\mathrm{Fr}$ \\
\hline Tecoma stans (L.) Juss. ex Kunth & candox & MCGR 47 & $b, e, g, h, i, j$ & $\begin{array}{c}21,22,24,37 \\
50,63\end{array}$ & 0.25 & 3.40 & 2.83 & 4.87 & 2.50 \\
\hline \multicolumn{10}{|l|}{ BIXACEAE } \\
\hline $\begin{array}{l}\text { Cochlospermum vitifolium (Willd.) } \\
\text { Spreng. } \\
\text { BORAGINACEAE }\end{array}$ & $\begin{array}{l}\text { pomposhuti, } \\
\text { tamborcito }\end{array}$ & MCGR 170 & $\mathrm{c}, \mathrm{g}, \mathrm{i}, \mathrm{j}$ & $\begin{array}{c}8,38,44,46 \\
47,50\end{array}$ & 0.15 & 1.95 & 1.93 & 2.50 & 1.43 \\
\hline Bourreria andrieuxii Hemsl. & yoa & MCGR 164 & $b, i$ & 66,76 & 0.01 & 1.58 & 1.32 & 1.62 & 1.79 \\
\hline $\begin{array}{l}\text { Cordia alba (Jacq.) Roem. \& } \\
\text { Schult. }\end{array}$ & matzú & $M C G R 22$ & $b, f, j$ & $\begin{array}{c}6,28,48,50 \\
55\end{array}$ & 0.04 & 1.14 & 1.30 & 0.87 & 1.25 \\
\hline $\begin{array}{l}\text { Cordia alliodora (Ruiz \& Pav.) } \\
\text { Oken }\end{array}$ & $\begin{array}{l}\text { palo de pajarito, } \\
\text { hormiguillo }\end{array}$ & $D A P M 66$ & $\begin{array}{l}\mathrm{b}, \mathrm{c}, \mathrm{f}, \mathrm{g} \\
\mathrm{h}, \mathrm{i}, \mathrm{j}\end{array}$ & $\begin{array}{l}9,14,29,50 \\
54,56,65,69 \\
71,76\end{array}$ & 0.40 & 0.68 & 0.47 & 0.67 & 0.89 \\
\hline Cordia dodecandra DC. & cupapé & MCGR 153 & $\begin{array}{c}a, b, c, f, h \\
i, j\end{array}$ & $\begin{array}{l}9,54,50,55 \\
65\end{array}$ & 0.12 & 0.90 & 1.07 & 0.55 & 1.07 \\
\hline \multicolumn{10}{|l|}{ BURSERACEAE } \\
\hline $\begin{array}{l}\text { Bursera bipinnata (Moc. \& Sessé } \\
\text { ex DC.) Engl. }\end{array}$ & $\begin{array}{l}\text { copal hoja } \\
\text { menuda }\end{array}$ & MCGR 179 & $\mathrm{~d}, \mathrm{f}, \mathrm{h}, \mathrm{i}, \mathrm{j}$ & $\begin{array}{c}4,12,29,50 \\
77\end{array}$ & 0.06 & 0.19 & 0.28 & 0.12 & 0.18 \\
\hline Bursera excelsa (Kunth) Engl. & $\begin{array}{l}\text { copal, copal de } \\
\text { zope }\end{array}$ & DAPM 91 & $\begin{array}{l}\mathrm{b}, \mathrm{c}, \mathrm{d}, \mathrm{e}, \mathrm{f} \\
\mathrm{g}, \mathrm{h}, \mathrm{i}, \mathrm{j}\end{array}$ & $\begin{array}{l}4,10,12,29 \\
37,40,45,50 \\
72,77\end{array}$ & 0.43 & 1.38 & 2.47 & 0.59 & 1.07 \\
\hline Bursera simaruba (L.) Sarg. & mulato & MCGR 58 & $\begin{array}{l}\mathrm{b}, \mathrm{c}, \mathrm{e}, \mathrm{g} \\
\mathrm{h}, \mathrm{i}, \mathrm{j}\end{array}$ & $\begin{array}{c}1,8,10,12, \\
13,17,20,22, \\
24,30,33,34, \\
37,43,44,46, \\
47,50,57\end{array}$ & 0.66 & 3.42 & 4.50 & 2.73 & 3.04 \\
\hline \multicolumn{10}{|l|}{ CELASTRACEAE } \\
\hline $\begin{array}{l}\text { Semialarium mexicanum (Miers) } \\
\text { Mennega } \\
\text { COMPOSITAE }\end{array}$ & matapiojo & MCGR 127 & $\mathrm{~J}$ & 50 & 0.01 & 0.23 & 0.22 & 0.28 & 0.18 \\
\hline $\begin{array}{l}\text { Ageratina ligustrina (DC.) R.M. } \\
\text { King \& H. Rob. }\end{array}$ & $\begin{array}{l}\text { flor blanca } \\
\text { cimarrona }\end{array}$ & MCGR 200 & $\mathrm{~b}, \mathrm{i}$ & 56,66 & 0.05 & - & - & - & - \\
\hline Montanoa tomentosa Cerv. & malacate & MCGR 109 & $b, f, h, i, j$ & $36,45,50,69$ & 0.08 & 3.56 & 3.59 & 5.11 & 1.96 \\
\hline Perymenium grande Hemsl. & malacate rojo & MCGR 119 & $\mathrm{~b}, \mathrm{~d}, \mathrm{f}, \mathrm{h}, \mathrm{i}, \mathrm{j}$ & $\begin{array}{c}17,49,50,60 \\
66,64\end{array}$ & 0.01 & 2.54 & 1.75 & 4.63 & 1.25 \\
\hline $\begin{array}{l}\text { Verbesina myriocephala Sch. Bip. } \\
\text { ex Klatt } \\
\text { EBENACEAE }\end{array}$ & bordón de viejo & DYSM 695 & b & 64 & 0.05 & - & - & - & - \\
\hline Diospyros nigra (J.F. Gmel.) Perrier & zapote negro & MCGR 173 & $\mathrm{a}, \mathrm{d}, \mathrm{f}, \mathrm{h}, \mathrm{i}, \mathrm{j}$ & $\begin{array}{c}5,35,50,67 \\
77\end{array}$ & 0.23 & 0.27 & 0.32 & 0.12 & 0.36 \\
\hline $\begin{array}{l}\text { Diospyros verae-crucis (Standl.) } \\
\text { Standl. } \\
\text { ERYTHROXYLACEAE }\end{array}$ & zapotillo & DYSM 65 & a & 35 & 0.01 & - & - & - & - \\
\hline Erythroxylum havanense Jacq. & escobillo & $M G R 163$ & $b, f, g, i, j$ & $17,31,50$ & 0.04 & 1.36 & 0.89 & 1.39 & 1.79 \\
\hline
\end{tabular}


Apéndice: Continuación.

\begin{tabular}{|c|c|c|c|c|c|c|c|c|c|}
\hline Familia/especie & $\begin{array}{l}\text { Nombre } \\
\text { común }\end{array}$ & No. Colecta & Usos & $\begin{array}{c}\text { Usos } \\
\text { específicos }\end{array}$ & VU & IVI & Do & De & Fr \\
\hline \multicolumn{10}{|l|}{ EUPHORBIACEAE } \\
\hline $\begin{array}{l}\text { Cnidoscolus aconitifolius (Mill.) } \\
\text { I.M. Johnst. }\end{array}$ & $\begin{array}{l}\text { chaya, chaya de } \\
\text { castilla }\end{array}$ & $M C G R 11$ & $\mathrm{e}, \mathrm{g}, \mathrm{h}$ & 37 & 0.03 & 1.29 & 0.86 & 1.23 & 1.79 \\
\hline Croton guatemalensis Lotsy & copalchi & MCGR 171 & $\mathrm{~b}, \mathrm{~g}, \mathrm{i}, \mathrm{j}$ & 45,50 & 0.13 & 0.16 & 0.09 & 0.20 & 0.18 \\
\hline Croton niveus Jacq. & $\begin{array}{l}\text { copalchi hoja } \\
\text { menuda }\end{array}$ & $D A P M 101$ & g & 38,39 & 0.01 & 0.39 & 0.44 & 0.36 & 0.36 \\
\hline Euphorbia leucocephala Lotsy & $\begin{array}{l}\text { flor de lechita, } \\
\text { punupupú }\end{array}$ & MCGR 196 & $\mathrm{~d}, \mathrm{~h}$ & 0 & 0.03 & - & - & - & - \\
\hline \multicolumn{10}{|l|}{ FABACEAE } \\
\hline Acacia collinsii Saff. & $\begin{array}{l}\text { iscanal, espina } \\
\text { de cacho }\end{array}$ & DYSM 716 & $e, f, h, i, j$ & 37,50 & 0.10 & - & - & - & - \\
\hline Acacia cornigera (L.) Willd. & cacho de toro & $M C G R 4$ & $\mathrm{~b}, \mathrm{f}, \mathrm{i}, \mathrm{j}$ & $\begin{array}{l}29,37,45 \\
50,56\end{array}$ & 0.18 & 0.85 & 0.38 & 0.55 & 1.61 \\
\hline Acacia farnesiana (L.) Willd. & huizache & DYSM 717 & $e, j$ & 37,50 & 0.04 & - & - & - & - \\
\hline Acacia pennatula (Schltdl. \& & quebracho & $M C G R 165$ & $\mathrm{~b}, \mathrm{c}, \mathrm{e}, \mathrm{i}, \mathrm{j}$ & 37,50 & 1.29 & 0.70 & 0.80 & 0.40 & 0.89 \\
\hline $\begin{array}{l}\text { Cham.) Benth. } \\
\text { Albizia lebbeck (L.) Benth. }\end{array}$ & guaje lanudo & MCGR 13 & $\mathrm{~b}$ & $45,56,69$ & 0.01 & 0.68 & 0.45 & 0.87 & 0.71 \\
\hline Albizia tomentosa (Micheli) Standl. & guanacastillo & MCGR 168 & $\mathrm{~b}$ & & 0.01 & 0.70 & 1.77 & 0.16 & 0.18 \\
\hline Bauhinia divaricata $\mathrm{L}$. & $\begin{array}{l}\text { casquito de } \\
\text { venado }\end{array}$ & $D A P M 117$ & $\mathrm{~g}, \mathrm{i}, \mathrm{j}$ & 17,50 & 0.07 & 1.47 & 1.61 & 1.74 & 1.07 \\
\hline Calliandra bijuga Rose & siete pellejos & DYSM 667 & $\mathrm{~d}, \mathrm{~h}, \mathrm{j}$ & 50 & 0.01 & - & - & - & - \\
\hline $\begin{array}{l}\text { Calliandra houstoniana (Mill.) } \\
\text { Standl. }\end{array}$ & palo de sangre & MCGR 21 & $\mathrm{i}$ & - & 0.01 & 0.57 & 0.36 & 0.63 & 0.71 \\
\hline $\begin{array}{l}\text { Diphysa americana (Mill.) M. } \\
\text { Sousa }\end{array}$ & guachipilin & MCGR 195 & $\mathrm{~b}, \mathrm{c}, \mathrm{f}, \mathrm{g}, \mathrm{i}, \mathrm{j}$ & $\begin{array}{l}50,53,59 \\
70,71\end{array}$ & 0.12 & - & - & - & - \\
\hline Erythrina americana Miller & frijolito & MCGR 197 & $\mathrm{a}, \mathrm{c}, \mathrm{e}, \mathrm{i}, \mathrm{j}$ & $34,37,41,50$ & 0.12 & - & - & - & - \\
\hline Erythrina goldmanii Standl. & $\begin{array}{c}\text { palo de } \\
\text { machetillo }\end{array}$ & DYSM 443 & $\mathrm{~b}$ & & 0.01 & - & - & - & - \\
\hline Eysenhardtia adenostylis Baill. & taray, palo dulce & MCGR 172 & $\begin{array}{l}a, b, c, d, e \\
f, g, h, i, j\end{array}$ & $\begin{array}{c}0,2,8,18,21 \\
22,36,37,38 \\
45,46,50,56 \\
66,77\end{array}$ & 1.36 & 0.28 & 0.19 & 0.28 & 0.36 \\
\hline Gliricidia sepium (Jacq.) Kunth & $\begin{array}{l}\text { mataratón, } \\
\text { madrecaco }\end{array}$ & MCGR 37 & $\begin{array}{c}\mathrm{a}, \mathrm{b}, \mathrm{c}, \mathrm{d}, \mathrm{f} \\
\mathrm{g}, \mathrm{h}, \mathrm{i}, \mathrm{j}\end{array}$ & $\begin{array}{c}0,11,29,45, \\
51,50,52,53, \\
54,56,59,62, \\
65,68,69, \\
70,77\end{array}$ & 0.48 & 0.21 & 0.06 & 0.20 & 0.36 \\
\hline Haematoxylum brasiletto H. Karst. & brasil & DYSM 547 & $\mathrm{~b}, \mathrm{f}, \mathrm{g}, \mathrm{i}, \mathrm{j}$ & $\begin{array}{c}2,8,18,19 \\
21,45,50,66\end{array}$ & 0.46 & - & - & - & - \\
\hline Leucaena collinsii Britton \& Rose & guaje & MCGR 183 & $\begin{array}{c}a, b, c, f, g \\
h, i, j\end{array}$ & $\begin{array}{c}35,45,50,54 \\
56,57,65 \\
66,77\end{array}$ & 0.97 & 0.59 & 0.66 & 0.75 & 0.36 \\
\hline
\end{tabular}


Apéndice: Continuación.

\begin{tabular}{|c|c|c|c|c|c|c|c|c|c|}
\hline Familia/especie & $\begin{array}{l}\text { Nombre } \\
\text { común }\end{array}$ & No. Colecta & Usos & $\begin{array}{c}\text { Usos } \\
\text { específicos }\end{array}$ & VU & IVI & Do & De & Fr \\
\hline $\begin{array}{l}\text { Leucaena diversifolia (Schltdl.) } \\
\text { Benth. }\end{array}$ & $\begin{array}{l}\text { guaje de } \\
\text { montaña }\end{array}$ & MCGR 175 & $a, b, g, i, j$ & $45,50,56,57$ & 0.10 & 0.24 & 0.24 & 0.12 & 0.36 \\
\hline $\begin{array}{l}\text { Leucaena leucocephala (Lam.) de } \\
\text { Wit }\end{array}$ & guaje blanco & MCGR 14 & $b, j$ & 50 & 0.04 & 0.10 & 0.07 & 0.04 & 0.18 \\
\hline Lonchocarpus guatemalensis Benth. & chaperla & MCGR 79 & $b, f, i, j$ & $\begin{array}{l}29,45,50,56 \\
\quad 66,69,75\end{array}$ & 0.12 & 0.65 & 0.55 & 1.03 & 0.36 \\
\hline Lonchocarpus rugosus Benth. & matabuey & DAPM 129 & $b, f, i, j$ & $\begin{array}{c}29,50,56,69 \\
70,77\end{array}$ & 0.14 & 2.47 & 0.81 & 0.59 & 1.07 \\
\hline $\begin{array}{l}\text { Lysiloma acapulcense (Kunth) } \\
\text { Benth. }\end{array}$ & tepehuaje & $D A P M 87$ & $\mathrm{~b}, \mathrm{f}, \mathrm{g}, \mathrm{i}, \mathrm{j}$ & $\begin{array}{c}45,50,53,56 \\
66,69,77\end{array}$ & 0.47 & 2.88 & 3.70 & 2.97 & 1.96 \\
\hline $\begin{array}{l}\text { Machaerium arboreum (Jacq.) } \\
\text { Vogel }\end{array}$ & $\begin{array}{c}\text { palo de } \\
\text { matagusano }\end{array}$ & MCGR 162 & $b, i, j$ & 50 & 0.03 & 2.81 & 2.42 & 4.04 & 1.96 \\
\hline $\begin{array}{l}\text { Mimosa zimapanensis Britton \& } \\
\text { Rose }\end{array}$ & tepezcouite & Reg. fot. & $\mathrm{g}, \mathrm{i}, \mathrm{j}$ & 50 & 0.03 & - & - & - & - \\
\hline Mimosa sp. 1 & sierrita & DYSM 495 & $b, e, f, i, j$ & $6,37,50,76$ & 0.28 & - & - & - & - \\
\hline Mimosa sp. 2 & amolillo & MCGR 193 & $\mathrm{~b}, \mathrm{i}, \mathrm{j}$ & $45,50,56,69$ & 0.21 & - & - & - & - \\
\hline Myroxylon balsamum (L.) Harms & palo de bálsamo & Reg. fot. & $b, f, g$ & 46 & 0.04 & - & - & - & - \\
\hline Pithecellobium pachypus Pittier & patzagua & DYSM 96 & $\mathrm{a}, \mathrm{i}, \mathrm{j}$ & 35,50 & 0.03 & & & & \\
\hline $\begin{array}{l}\text { Senna nicaraguensis (Benth.) H.S. } \\
\text { Irwin \& Barneby }\end{array}$ & huevo de iguana & MCGR 176 & $\mathrm{~h}$ & & 0.01 & 0.23 & 0.09 & 0.24 & 0.36 \\
\hline $\begin{array}{l}\text { Senna skinneri (Benth.) H.S. Irwin } \\
\text { \& Barneby } \\
\text { HERNANDIACEAE }\end{array}$ & Santa rosa & $D A P M 125$ & $b, f, h, i, j$ & 50 & 0.22 & 0.11 & 0.08 & 0.08 & 0.18 \\
\hline Gyrocarpus mocinoi Espejo & San Felipe & MCGR 155 & $b, f, i, j$ & $\begin{array}{l}9,50,54,60 \\
65\end{array}$ & 0.07 & 0.19 & 0.23 & 0.16 & 0.18 \\
\hline \multicolumn{10}{|l|}{ LAURACEAE } \\
\hline Litsea glaucescens Kunth & laurel & Reg. fot. & a & 16 & 0.02 & - & - & - & - \\
\hline Persea americana Mill. & aguacate & MCGR 204 & $a, b, g, i, j$ & $\begin{array}{l}15,16,20,22 \\
35,45,50\end{array}$ & 0.39 & - & - & - & - \\
\hline \multicolumn{10}{|l|}{ MALPIGHIACEAE } \\
\hline Byrsonima crassifolia (L.) Kunth & nanche & MCGR 169 & $\begin{array}{c}a, b, c, e, g \\
h, i, j\end{array}$ & $\begin{array}{l}26,35,34,37 \\
39,50\end{array}$ & 0.35 & 0.35 & 0.38 & 0.32 & 0.36 \\
\hline \multicolumn{10}{|l|}{ MALVACEAE } \\
\hline $\begin{array}{l}\text { Ceiba aesculifolia (Kunth) Britten } \\
\text { \& Baker f. }\end{array}$ & mosmoté & DAPM 16 & $b, e, f, i, j$ & $\begin{array}{c}7,12,34,37 \\
50,77\end{array}$ & 0.16 & 0.64 & 0.55 & 0.48 & 0.89 \\
\hline Guazuma ulmifolia Lam. & cuahulote & MCGR 181 & $\begin{array}{c}a, b, e, f, g \\
h, i, j\end{array}$ & $\begin{array}{l}6,12,22,34 \\
35,37,49,50\end{array}$ & 0.26 & 0.34 & 0.30 & 0.36 & 0.36 \\
\hline Hampea mexicana Fryxell & majagua & $D A P M 516$ & $\mathrm{~b}$ & 45 & 0.02 & - & - & - & - \\
\hline $\begin{array}{l}\text { Heliocarpus terebinthinaceus (DC.) } \\
\text { Hochr. }\end{array}$ & guaspó, corcho & MCGR 37 & $b, f, i, j$ & $\begin{array}{l}36,50,56,66 \\
69,73,76\end{array}$ & 0.27 & 2.94 & 3.82 & 3.21 & 1.79 \\
\hline $\begin{array}{l}\text { Luehea candida (Moc. \& Sessé ex } \\
\text { DC.) Mart. }\end{array}$ & cascabillo & MCGR 182 & $\mathrm{~b}, \mathrm{c}, \mathrm{g}, \mathrm{i}, \mathrm{j}$ & 50,61 & 0.12 & 3.88 & 7.88 & 2.69 & 1.07 \\
\hline
\end{tabular}


Apéndice: Continuación.

\begin{tabular}{|c|c|c|c|c|c|c|c|c|c|}
\hline Familia/especie & $\begin{array}{l}\text { Nombre } \\
\text { común }\end{array}$ & No. Colecta & Usos & $\begin{array}{c}\text { Usos } \\
\text { específicos }\end{array}$ & VU & IVI & Do & De & $\mathrm{Fr}$ \\
\hline \multicolumn{10}{|l|}{ MELIACEAE } \\
\hline Cedrela odorata L. & cedro & MCGR 191 & $\begin{array}{l}\mathrm{b}, \mathrm{c}, \mathrm{f}, \mathrm{g} \\
\mathrm{h}, \mathrm{i}, \mathrm{j}\end{array}$ & $\begin{array}{c}9,50,51,54 \\
55,65,77\end{array}$ & 0.66 & 0.08 & 0.01 & 0.04 & 0.18 \\
\hline Cedrela tonduzii C. DC. & cedril & $D A P M 31$ & $\mathrm{f}$ & & 0.01 & 0.08 & 0.01 & 0.04 & 0.18 \\
\hline Swietenia humilis Zucc. & caoba & $M C G R 205$ & $\begin{array}{l}\mathrm{b}, \mathrm{c}, \mathrm{f}, \mathrm{g} \\
\mathrm{h}, \mathrm{i}, \mathrm{j}\end{array}$ & $\begin{array}{c}9,21,29,32 \\
45,50,51,54 \\
55,58,62 \\
65,77\end{array}$ & 0.56 & - & - & - & - \\
\hline Trichilia hirta L. & napaguito & $D A P M 154$ & $b, f$ & $54,56,65,69$ & 0.02 & 0.37 & 0.16 & 0.24 & 0.71 \\
\hline MORACEAE & & & & & 0.66 & 0.08 & 0.01 & 0.04 & 0.18 \\
\hline Ficus aurea Nutt. & palo de higo & $M C G R 113$ & $\begin{array}{l}\mathrm{b}, \mathrm{c}, \mathrm{f}, \mathrm{g} \\
\mathrm{h}, \mathrm{i}, \mathrm{j}\end{array}$ & 12,50 & 0.18 & 1.23 & 1.76 & 0.67 & 1.25 \\
\hline \multicolumn{10}{|l|}{ MUNTINGIACEAE } \\
\hline Muntingia calabura L. & capulín & MCGR 199 & $\mathrm{a}, \mathrm{i}, \mathrm{j}$ & 50 & 0.04 & - & - & - & - \\
\hline \multicolumn{10}{|l|}{ MYRTACEAE } \\
\hline Eugenia breedlovei Barrie & chazá & $D A P M 138$ & $b, \mathrm{i}, \mathrm{j}$ & $0,45,50,56$ & 0.07 & 0.70 & 0.85 & 0.71 & 0.54 \\
\hline $\begin{array}{l}\text { Eugenia capuli (Schltdl. \& Cham.) } \\
\text { Hook. \& Arn. }\end{array}$ & guayabillo & $D A P M 110$ & $\mathrm{~b}$ & 56,69 & 0.01 & 0.80 & 0.32 & 0.83 & 1.25 \\
\hline Psidium guajava $\mathrm{L}$. & guayaba & MCGR 201 & $a, b, f, g, i, j$ & $\begin{array}{l}22,24,33 \\
\quad 35,50\end{array}$ & 0.12 & - & - & - & - \\
\hline $\begin{array}{l}\text { Psidium sartorianum (O. Berg) } \\
\text { Nied. } \\
\text { OLACACEAE }\end{array}$ & guayabillo & $D A P M 421$ & $\mathrm{a}$ & 35 & 0.01 & - & - & - & - \\
\hline $\begin{array}{l}\text { Ximenia americana } \mathrm{L} \text {. } \\
\text { OLEACEAE }\end{array}$ & \multicolumn{8}{|c|}{ OLEACEAE } & 0.36 \\
\hline Fraxinus purpusii Brandegee & $\begin{array}{l}\text { saucito, } \\
\text { botásvaras }\end{array}$ & $D A P M 130$ & $\begin{array}{c}b, c, e, f, g \\
i, j\end{array}$ & $\begin{array}{l}7,37,45,50 \\
56,66,77,71\end{array}$ & 0.35 & 1.54 & 2.20 & 0.63 & 1.79 \\
\hline \multicolumn{10}{|l|}{ POLYGONACEAE } \\
\hline $\begin{array}{l}\text { Gymnopodium floribundum Rolfe. } \\
\text { PRICAMIACEAE }\end{array}$ & aguanatocal & MCGR 69 & $b, j$ & 50 & 0.07 & 1.19 & 0.80 & 1.70 & 1.07 \\
\hline Alvaradoa amorphoides Liebm. & camarón & $D A P M 103$ & $\mathrm{~b}, \mathrm{c}, \mathrm{f}, \mathrm{g}, \mathrm{i}, \mathrm{j}$ & $\begin{array}{c}23,39,50,56 \\
66,76\end{array}$ & 0.48 & 1.96 & 2.49 & 1.43 & 1.96 \\
\hline \multicolumn{10}{|l|}{ PRIMULACEAE } \\
\hline $\begin{array}{l}\text { Bonellia macrocarpa (Cav.) B. } \\
\text { Ståhl \& Källersjö } \\
\text { RHAMNACEAE }\end{array}$ & sicqueté & DAPM 296 & $\mathrm{~d}, \mathrm{~h}, \mathrm{i}, \mathrm{j}$ & 3,50 & 0.04 & 0.91 & 0.61 & 0.87 & 1.25 \\
\hline Colubrina arborescens Sarg. & cascarillo & $D A P M 44$ & $\mathrm{~b}, \mathrm{c}, \mathrm{f}, \mathrm{g}, \mathrm{i}, \mathrm{j}$ & $\begin{array}{c}6,21,24,36 \\
45,50,54,56 \\
64,65,66 \\
69,77\end{array}$ & 0.89 & - & - & - & - \\
\hline
\end{tabular}


Apéndice: Continuación.

\begin{tabular}{|c|c|c|c|c|c|c|c|c|c|}
\hline Familia/especie & $\begin{array}{l}\text { Nombre } \\
\text { común }\end{array}$ & No. Colecta & Usos & $\begin{array}{c}\text { Usos } \\
\text { específicos }\end{array}$ & $\mathrm{VU}$ & IVI & Do & $\mathrm{De}$ & $\mathrm{Fr}$ \\
\hline $\begin{array}{l}\text { Colubrina triflora Brongn. ex G. } \\
\text { Don } \\
\text { RUBIACEAE }\end{array}$ & corazón bonito & MCGR 33 & $\mathrm{~b}, \mathrm{c}, \mathrm{g}, \mathrm{i}$ & - & 0.04 & 1.43 & 1.63 & 1.58 & 1.07 \\
\hline $\begin{array}{l}\text { Calycophyllum candidissimum } \\
\text { (Vahl) DC. }\end{array}$ & palo canelo & DAPM 39 & $b, g, i, j$ & 45,50 & 0.12 & 0.08 & 0.01 & 0.04 & 0.18 \\
\hline Hamelia patens Jacq. & coralillo & $D A P M 29$ & g & 38,74 & 0.04 & 0.38 & 0.61 & 0.16 & 0.36 \\
\hline $\begin{array}{l}\text { Randia micracantha (Lillo) } \\
\text { Bacigalupo } \\
\text { RUTACEAE }\end{array}$ & rompezapato & MCGR 139 & $\mathrm{i}, \mathrm{j}$ & 50 & 0.12 & 0.33 & 0.09 & 0.20 & 0.71 \\
\hline $\begin{array}{l}\text { Zanthoxylum fagara (L.) Sarg. } \\
\text { SALICACEAE }\end{array}$ & palo de alacrán & $D A P M 40$ & $\mathrm{~g}, \mathrm{j}$ & 26,50 & 0.04 & 0.80 & 0.60 & 0.91 & 0.89 \\
\hline Prockia crucis P. Browne ex L. & palo de piedra & $D A P M 179$ & $\mathrm{~b}$ & - & 0.01 & 0.29 & 0.30 & 0.20 & 0.36 \\
\hline $\begin{array}{l}\text { Zuelania guidonia (Sw.) Britton \& } \\
\text { Millsp. } \\
\text { SAPOTACEAE }\end{array}$ & paraguita & DYSM 04 & $\mathrm{~b}, \mathrm{~h}, \mathrm{i}, \mathrm{j}$ & $50,56,66,76$ & 0.03 & - & - & - & - \\
\hline $\begin{array}{l}\text { Sideroxylon capiri (A. DC.) Pittier } \\
\text { SIMAROUBACEAE }\end{array}$ & tempizque & DYSM 197 & $\mathrm{~d}, \mathrm{e}, \mathrm{h}, \mathrm{i}, \mathrm{j}$ & $34,37,50,67$ & 0.22 & 0.18 & 0.07 & 0.12 & 0.36 \\
\hline $\begin{array}{l}\text { Simarouba amara Aubl. } \\
\text { URTICACEAE }\end{array}$ & aceituna & DYSM 156 & $a, b, e, f, i$ & $29,34,37$ & 0.12 & - & - & - & - \\
\hline $\begin{array}{l}\text { Cecropia obtusifolia Bertol. } \\
\text { VERBENACEAE }\end{array}$ & guarumbo & MCGR 185 & $\mathrm{~g}, \mathrm{~h}, \mathrm{j}$ & $50,24,47$ & 0.07 & 0.08 & 0.02 & 0.04 & 0.18 \\
\hline $\begin{array}{l}\text { Lippia chiapasensis Loes. } \\
\text { ZYGOPHYLLACEAE }\end{array}$ & guayabita agria & $D A P M 75$ & g & - & 0.02 & 0.64 & 0.32 & 0.71 & 0.89 \\
\hline Guaiacum sanctum L. & guayacán & DYSM 669 & $b, i, j$ & 50 & 0.03 & - & - & - & - \\
\hline
\end{tabular}

